\title{
Hilbert Functions of Fat Point Subschemes of the Plane: the Two-fold Way
}

\author{
Anthony V. Geramita, Brian Harbourne, and Juan C. Migliore
}

\begin{abstract}
Two approaches for determining Hilbert functions of fat point subschemes of $\mathbb{P}^{2}$ are demonstrated. A complete determination of the Hilbert functions which occur for 9 double points is given using the first approach, extending results obtained in a previous paper using the second approach. In addition the second approach is used to obtain a complete determination of the Hilbert functions for $n \geq 9 m$-multiple points for every $m$ if the points are smooth points of an irreducible plane cubic curve. Additional results are obtained using the first approach for $n \geq 9$ double points when the points lie on an irreducible cubic (but now are not assumed to be smooth points of the cubic).
\end{abstract}

Keywords. Fat Points, Hilbert Functions, Linkage, Blow Ups, Projective Plane.

2010 Mathematics Subject Classification. Primary: 13D40, 14J26, 14M07; Secondary: 14M06, 14N05.

\section{Introduction}

If $X$ is a reduced set of $n$ points in $\mathbb{P}^{2}$, the fat point subscheme $Z=m X \subset \mathbb{P}^{2}$ is the $(m-1)$-st infinitesimal neighborhood of $X$. Thus $m X$ is the subscheme defined by the symbolic power $I(X)^{(m)} \subset R=k\left[\mathbb{P}^{2}\right]$ (that is, by the saturation of the ideal $I(X)^{m}$ with respect to the ideal generated by the coordinate variables in the ring $\left.k\left[\mathbb{P}^{2}\right]\right)$. The question motivating this paper is: What are the Hilbert functions of such subschemes of $\mathbb{P}^{2}$ ? There have been two main approaches to this question, and one goal of this paper is to demonstrate them in various situations.

The two approaches are exemplified by the papers [9] and [7]. The approach of [9] is to identify constraints that Hilbert functions must satisfy and then for each function satisfying those constraints to try to find a specific subscheme having that function as its Hilbert function. A complete classification of all Hilbert functions of reduced 0-dimensional subschemes of projective space was given in [8] using essentially this approach. The paper [9] then uses [8] as the starting point for classifying Hilbert functions for subschemes of the form $Z=2 X \subset \mathbb{P}^{2}$ with $X$ reduced and 0-dimensional.

We thank the NSF, whose support for the MAGIC05 conference at the University of Notre Dame gave us an opportunity to begin work on this paper. Geramita also thanks the NSERC for research support, and Harbourne and Migliore thank the NSA for its partial support of their research (under grants H9823011-1-0139 and H98230-07-1-0066 for Harbourne and under grants H98230-07-1-0036 and H98230-091-0031 for Migliore). 
This approach is most effective when the class of possible functions is fairly limited, hence the restriction in [9] to the case $m=2$. This approach has the advantage of providing explicit results often without needing detailed information about the disposition of the points, but it has the disadvantage of not providing a complete dictionary of which point sets give which Hilbert function. The approach of [7] is to use the geometry of the surface $Y$ obtained by blowing up the points of the support of $Z$ to obtain information about the Hilbert function of $Z$. This approach is most effective when the geometry of $Y$ is well-understood, hence the restriction in [7] to the case $n \leq 8$. Given points $p_{i}$ and non-negative integers $m_{i}$, the subscheme defined by the ideal $\bigcap_{i=1}^{n}\left(I\left(p_{i}\right)^{m_{i}}\right)$ is also called a fat point subscheme, and is denoted by $m_{1} p_{1}+\cdots+m_{n} p_{n}$. The advantage of the second approach, as implemented in [7], is that it provided complete results for all fat point subschemes $Z=m_{1} p_{1}+\cdots+m_{n} p_{n}$ with $n \leq 8$, together with a complete determination of which $Z$ give the same Hilbert function, but it had the cost of needing a lengthy analysis of the geometry of $Y$, and gives only recursive determinations of the Hilbert functions. However, for $n \leq 8$ and $k=2$ there are only finitely many cases, so a complete list of the Hilbert functions which occur can be given. See [7] for this list.

The first case left open by [7] is $n=9$ points of $\mathbb{P}^{2}$. It should, in principle, be possible to carry out the necessary analysis to obtain a complete recursive classification of Hilbert functions and corresponding points sets for $n=9$, but whereas for $n \leq 8$ there are only finitely many classes of sets of $n$ points, there will certainly be infinitely many when $n=9$ (related to the fact that there can be infinitely many prime divisors on $Y$ of negative self-intersection, and to the fact that effective nef divisors $F$ can occur with $\left.h^{1}\left(Y, \mathcal{O}_{Y}(F)\right)>0\right)$. Thus a complete classification in this case using the methods of [7] will be a substantial effort, which we leave for future research (not necessarily by us).

Instead, in this paper we will focus on some special cases. We devote Section 2 to demonstrating the first approach by obtaining a complete answer in the case of $n=9$ and $m=2$. This also shows how one could recover the result for $n=8$ and $m=2$ obtained in [7] using the methods of [9].

The rest of the paper is devoted to demonstrating both methods for the case of $n$ points of multiplicity $m$ on cubics, under somewhat different hypotheses chosen to play to the strengths of each method. The Philosophy of the First Way is to use known facts about Hilbert functions to say things about what Hilbert functions are possible. The Philosophy of the Second Way is to use known facts about cohomology of blown up surfaces to say things about what dimensions of linear systems are possible. Sections 3 (using the First Way) and 4 (using the Second Way) illustrate how we can attack the same problem and obtain overlapping and sometimes complementary results, but using dramatically different ways to do so.

So, given points on a plane cubic, for the First Way we will assume the cubic is irreducible, that $m=2$ and, in some cases, that $n$ is not too small. Our main results here are Theorem 3.4 and Theorem 3.7. For the Second Way we will make no re- 
strictions on $m$ nor assume the cubic is irreducible but we will assume the points are smooth points of the cubic and we will assume that the points are evenly distributed (meaning essentially that no component contains too many of the points). Under these two assumptions we give a complete determination of all possible Hilbert functions in Theorem 4.2. Using the same techniques we will, in Remark 4.5, also recover the Hilbert functions for $X$ and $2 X$ when $X$ is a reduced set of points contained in a reduced, irreducible singular cubic curve in case the singular point of the curve is one of the points of $X$.

We now discuss both methods in somewhat more detail. For the first approach we will follow [7] and [9] and sometimes work with the first difference, $\Delta h_{2 X}$, of the Hilbert function $h_{2 X}$ rather than with $h_{2 X}$ directly, since for our purposes $\Delta h_{2 X}$ is easier to work with. (For clarity we will refer to it as the Hilbert difference function, but we regard $\Delta h_{2 X}$ as just an equivalent formulation of the Hilbert function, so whenever our goal is to determine a Hilbert function, we will regard specifying the Hilbert difference function as having achieved the goal.) The first approach can be summarized as follows. We start by listing all Hilbert difference functions $\Delta h_{X}$ for reduced sets $X$ of $n=9$ points, using [8], and then we analyze each case in turn using $h_{X}$ to constrain the behavior of $h_{2 X}$. For example, in some extreme cases the form of $\Delta h_{X}$ forces many of the points of $X$ to lie on a line; knowing this can be very useful in determining $h_{2 X}$.

Our analysis uses the following tools: (i) a crude bound on the regularity of $I(2 X)$, giving an upper bound for the last degree in which $\Delta h_{2 X}$ can be non-zero; (ii) Bézout considerations giving the values of $\Delta h_{2 X}$ in most degrees; (iii) the fact that the sum of the values of $\Delta h_{2 X}$ is 27; and (iv) a theorem of Davis [4] giving geometric consequences for certain behavior of the function $\Delta h_{2 X}$. The idea is that we know the value of the Hilbert function for most degrees by (i), (ii) and (iii), and we can exhaustively list the possibilities for the remaining degrees. Then we use (iv) to rule out many of these. Finally, for the cases that remain, we try to construct examples of them (and in the situations studied in this paper, we succeed).

For the second approach we study $h_{Z}$ for an arbitrary fat point subscheme $Z=$ $m_{1} p_{1}+\cdots+m_{n} p_{n} \subset \mathbb{P}^{2}$ using the geometry of the surface $Y$, where $\pi: Y \rightarrow \mathbb{P}^{2}$ is the morphism obtained by blowing up the points $p_{i}$. This depends on the well known fact that $\operatorname{dim} I(Z)_{t}=h^{0}\left(Y, \mathcal{O}_{Y}(F)\right)$ where $F=t L-m_{1} E_{1}-\cdots-m_{n} E_{n}$, $\mathcal{O}_{Y}(L)=\pi^{*} \mathcal{O}_{\mathbb{P}^{2}}(1)$ and $E_{i}=\pi^{-1}\left(p_{i}\right)$. The fundamental fact here is the theorem of Riemann-Roch:

$$
\begin{aligned}
h^{0}\left(Y, \mathcal{O}_{Y}(F)\right) & -h^{1}\left(Y, \mathcal{O}_{Y}(F)\right)+h^{2}\left(Y, \mathcal{O}_{Y}(F)\right) \\
& =\frac{F^{2}-K_{Y} \cdot F}{2}+1=\left(\begin{array}{c}
t+2 \\
2
\end{array}\right)-\sum_{i}\left(\begin{array}{c}
m_{i}+1 \\
2
\end{array}\right) .
\end{aligned}
$$

To see the relevance of (1.1), note that $K_{Y}=-3 L+E_{1}+\cdots+E_{n}$, so we have by duality that $h^{2}\left(Y, \mathcal{O}_{Y}(F)\right)=h^{0}\left(Y, \mathcal{O}_{Y}\left(K_{Y}-F\right)\right)$ and thus $h^{2}\left(Y, \mathcal{O}_{Y}(F)\right)=0$ if 
$t>-3$. Now, since we are interested in the values of Hilbert functions when $t \geq 0$, we have $h_{Z}(t)=\operatorname{dim}\left(R_{t}\right)-\operatorname{dim}\left(I(Z)_{t}\right)=\left(\begin{array}{c}t+2 \\ 2\end{array}\right)-h^{0}\left(Y, \mathcal{O}_{Y}(F)\right)$ which using $(1.1)$ becomes

$$
h_{Z}(t)=\sum_{i}\left(\begin{array}{c}
m_{i}+1 \\
2
\end{array}\right)-h^{1}\left(Y, \mathcal{O}_{Y}(F)\right) .
$$

This second approach, as applied in [7], depended on knowing two things: the set $\operatorname{Neg}(Y)$ of all prime divisors $C$ on $Y$ with $C^{2}<0$ and on knowing $h^{0}\left(Y, \mathcal{O}_{Y}(F)\right)$ for every divisor $F$ for which we have $F \cdot C \geq 0$ for all $C \in \operatorname{Neg}(Y)$. $\operatorname{Given} \operatorname{Neg}(Y)$, one can in principle reduce the problem of computing $h^{0}\left(Y, \mathcal{O}_{Y}(F)\right)$ for an arbitrary divisor $F$ to the case that $F \cdot C \geq 0$ for all $C \in \operatorname{Neg}(Y)$. If $n \geq 2$ and $F \cdot C \geq 0$ for all $C \in \operatorname{Neg}(Y)$, then $h^{2}\left(Y, \mathcal{O}_{Y}(F)\right)=0$, so from Riemann-Roch we have only $h^{0}\left(Y, \mathcal{O}_{Y}(F)\right) \geq 1+\left(F^{2}-K_{Y} \cdot F\right) / 2$.

When $n \leq 8$ or the points $p_{i}$ lie on a conic (possibly singular), this inequality is always an equality, but for $n \geq 9$ points not contained in a conic it need not be, so more information in general is needed. Similarly, in case $n \leq 8$ or the points $p_{i}$ lie on a conic (possibly singular), it turns out, in fact, that $\operatorname{Neg}(Y)$ is a finite set, but this also can fail for $n \geq 9$ points not contained in a conic. As a consequence, given $\operatorname{Neg}(Y)$ one can determine $h_{Z}$ for any fat point subscheme $Z=m_{1} p_{1}+\cdots+m_{n} p_{n} \subset \mathbb{P}^{2}$ if either $n \leq 8$ or the points $p_{i}$ lie on a conic. This raises the question of what sets $\operatorname{Neg}(Y)$ occur under these assumptions. We answered this question in [7]. There are only finitely many possibilities and [7] gives a complete list.

When $n \geq 9$ and the points $p_{i}$ do not lie on a conic then not only can $\operatorname{Neg}(Y)$ fail to be finite but $h^{1}\left(Y, \mathcal{O}_{Y}(F)\right)$ need not vanish, even if $F \cdot C \geq 0$ for all $C \in \operatorname{Neg}(Y)$ and even if $F$ is effective. Assuming that the points $p_{i}$ lie on a cubic curve does not eliminate either difficulty, but it does mean that $-K_{Y}$ is effective (whether the cubic is irreducible or not), and thus the results of [12] can be applied to the problem of computing $h^{0}\left(Y, \mathcal{O}_{Y}(F)\right)$. In case $-K_{Y}$ is effective, it is known what kinds of classes can be elements of $\operatorname{Neg}(Y)$, but no one has yet classified precisely which sets $\operatorname{Neg}(Y)$ arise for $n \geq 9$ (doing this for $n=7,8$ was the new contribution in [7]). On the other hand, even without this complete classification, partial results can still be obtained using the second approach, as we will show here using information about the geometry of $Y$ developed in [12].

\section{Approach I: Nine Double Points}

It is natural to ask what can be said for fat point schemes $Z$ supported at $n>8$ points. As observed in [7, Remark 2.2], there are infinitely many configuration types of $n>8$ points, so we will restrict our attention to subschemes $2 Z=2\left(p_{1}+\cdots+p_{n}\right)$ of $\mathbb{P}^{2}$. Since we are now restricting the multiplicities of the points to be at most 2 , it is not necessary to make an exhaustive list of the configuration types - indeed, we will point out situations where different configurations exist but nevertheless do not give different 
Hilbert functions. Instead, in this situation we can bring to bear the methods developed in [9], and to demonstrate additional methods which can be used. We will determine all Hilbert functions that occur for double point subschemes $2 Z=2\left(p_{1}+\cdots+p_{9}\right)$ of $\mathbb{P}^{2}$, for every Hilbert function occurring as the Hilbert function of a simple point subscheme $Z=p_{1}+\cdots+p_{9}$.

Definition 2.1. Let $Z$ be a zero-dimensional subscheme of $\mathbb{P}^{N}$ with Hilbert function $h_{Z}$. The difference function of $Z$ is the first difference of the Hilbert function of $Z$, $\Delta h_{Z}(t)=h_{Z}(t)-h_{Z}(t-1)$. (This is sometimes also called the $h$-vector of $Z$, and sometimes the Castelnuovo function of $Z$.)

The Hilbert function and its difference function clearly give equivalent information and it is primarily because of the simpler bookkeeping allowed by the first difference that we use it. Notice that $\Delta h_{Z}$ is the Hilbert function of any Artinian reduction of $R / I_{Z}$ by a linear form.

One problem raised in [9] is the existence and determination of maximal and minimal Hilbert functions. In the current context, this means that we fix an underlying Hilbert function $\underline{h}$ that exists for some set of 9 points in $\mathbb{P}^{2}$, and letting $X$ move in the irreducible flat family of all sets of points with Hilbert function $\underline{h}$, we ask whether there is a maximal and a minimal Hilbert function for the corresponding schemes $Z=2 X$. It was shown in [9] that there does exist a maximal such Hilbert function, denoted $\underline{h}^{\max }$ (for any number of points). The proof in [9] is nonconstructive, and [9] determines $\underline{h}^{\max }$ in only a few special cases. The paper [9] also raises the question of whether $\underline{h}^{\text {min }}$ always exists; i.e., whether there exists an $X^{\prime}$ such that $h_{2 X}$ is at least as big in every degree as $h_{2 X^{\prime}}$ for every $X$ with $h_{X}=h_{X^{\prime}}$. This question remains open.

A useful tool is the following lemma. This lemma, and generalizations of it, are well-known. For a very short proof of the statement given here see [9, Lemma 2.18].

Lemma 2.2. Let $X$ be a reduced set of points in $\mathbb{P}^{2}$ with regularity $r+1$. Then the regularity of $I_{2 X}$ is bounded by $\operatorname{reg}\left(I_{2 X}\right) \leq 2 \cdot \operatorname{reg}\left(I_{X}\right)=2 r+2$.

We will also use the following result of Davis [4]. It is a special case of a more general phenomenon [1] related to maximal growth of the first difference of the Hilbert function.

Theorem 2.3. Let $X \subset \mathbb{P}^{2}$ be a zero-dimensional subscheme, and assume that $\Delta h_{X}(t)=\Delta h_{X}(t+1)=d$ for some $t, d$. Then the degree $t$ and the degree $t+1$ components of $I_{X}$ have a GCD, $F$, of degree $d$. Furthermore, the subscheme $W_{1}$ of $X$ lying on the curve defined by $F$ (i.e. $I_{W_{1}}$ is the saturation of the ideal $\left(I_{X}, F\right)$ ) has Hilbert function whose first difference is given by the truncation

$$
\Delta h_{W_{1}}(s)=\min \left\{\Delta h_{X}(s), d\right\} .
$$


Furthermore, the Hilbert function of the points $W_{2}$ not on $F$ (defined by $I_{W_{2}}=I_{X}$ : $(F))$ has first difference given by the (shifted) part above the truncation:

$$
\Delta h_{W_{2}}(s)=\max \left\{\Delta h_{X}(s+d)-d, 0\right\} .
$$

We will see precisely the possibilities that occur for the first infinitesimal neighborhood of nine points, and we will see that there is in each case a maximum and minimum Hilbert function. All together, there occur eight Hilbert functions for schemes $X=p_{1}+\cdots+p_{9}$. We give their difference functions, and the possible Hilbert functions that occur for double point schemes $2 X$, in the following theorem.

Theorem 2.4. The following table lists all possibilities for the Hilbert difference function for nine double points, in terms of the Hilbert difference function of the underlying nine points. In particular, for each Hilbert function $\underline{h}$, both $\underline{h}^{\max }$ and $\underline{h}^{\min }$ exist, and we indicate by "max" or "min" the function that achieves $\underline{h}^{\max }$ or $\underline{h}^{\min }$, respectively, for each $\underline{h}$. Of course when we have " $\max =$ min," the Hilbert function of $2 X$ is uniquely determined by that of $X$.

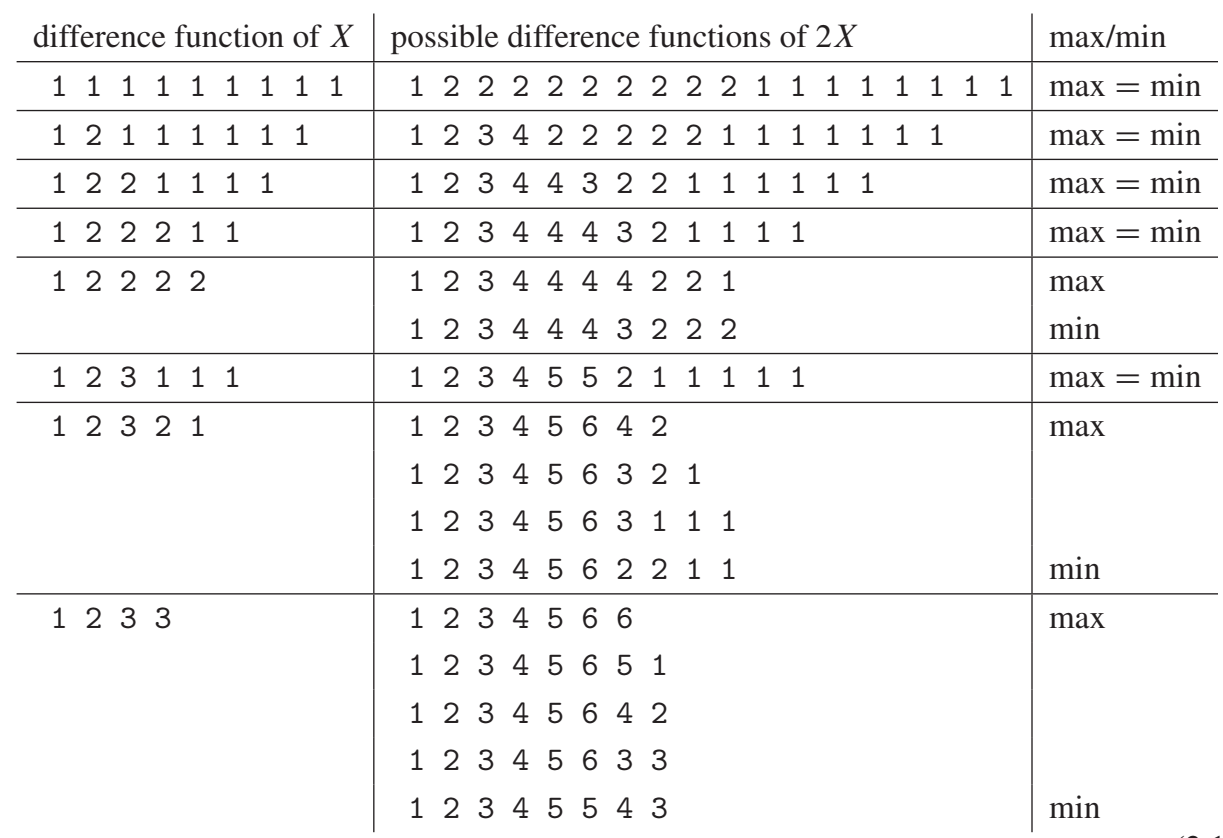

Proof. One has to "integrate" the difference functions in order to verify the claims about $\underline{h}^{\max }$ or $\underline{h}^{\min }$. We leave this to the reader. The fact that the eight Hilbert functions specified above for $X$ give a complete list is standard, and we omit the proof. 


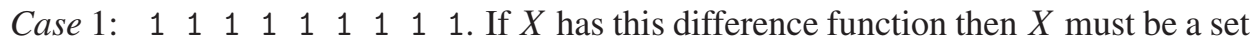
of 9 collinear points in $\mathbb{P}^{2}$. Such a set of points is necessarily a complete intersection, so it is easy to check that the difference function for $2 X$ is the one claimed. (Even the minimal free resolution is well known; see for instance $[2,3,10,11]$.)

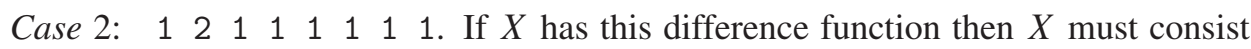
of 8 points on a line and one point off the line (it follows from Theorem 2.3). It is not hard to check, using Bézout arguments, that then $2 X$ has the claimed difference function.

Case 3: $\begin{array}{lllllll}1 & 2 & 2 & 1 & 1 & 1 & 1\end{array}$. If $X$ has this difference function then $X$ must consist of seven points on a line, say $\lambda_{1}$, and two points off the line (again using Theorem 2.3). Let $Q_{1}, Q_{2}$ be these latter points. We will see that the Hilbert function is independent of whether $Q_{1}$ and $Q_{2}$ are collinear with one of the seven other points or not. Note first that $2 X$ contains a subscheme of degree 14 lying on a line. Hence the regularity is $\geq 14$, so the difference function ends in degree $\geq 13$.

Let $L_{1}$ be a linear form defining $\lambda_{1}$ and let $L_{2}$ be a linear form defining the line joining $Q_{1}$ and $Q_{2}$. Using Bézout's theorem, it is clear that there is no form of degree $\leq 3$ vanishing on $2 X$. Furthermore, $L_{1}^{2} L_{2}^{2}$ is the only form (up to scalar multiples) of degree 4 vanishing on $2 X$. Now, in degrees 5,6 and 7 we have that $L_{1}^{2}$ is a common factor for all forms in the ideal of $2 X$. Hence $\left(I_{2} Q_{1}+2 Q_{2}\right)_{t-2} \cong\left(I_{2 X}\right)_{t}$ for $5 \leq t \leq 7$, where the isomorphism is obtained by multiplying by $L_{1}^{2}$. But $2 Q_{1}+2 Q_{2}$ imposes independent conditions on forms of degree 3 , so we can compute that $\operatorname{dim}\left(I_{2 X}\right)_{t}=$ $4,9,15$ for $t=5,6,7$ respectively.

The calculations above give the claimed difference function up to degree 7 , namely $(1,2,3,4,4,3,2,2)$. But the sum of the terms of the difference function has to equal $27(=\operatorname{deg} 2 X)$, and the terms past degree 7 must be non-increasing, and positive through degree 13 . This is enough to force the claimed difference function.

Case 4: 122221 1. By Theorem 2.3, $X$ must consist of six points, $X_{1}$, on a line, $\lambda_{1}$, and three collinear points, $X_{2}$, on another line, $\lambda_{2}$. The intersection of $\lambda_{1}$ and $\lambda_{2}$ may or may not be a point of $X_{1}$; it is not a point of $X_{2}$. We will see, as in Case 3, that this combinatorial distinction does not affect the Hilbert function of $2 X$. Pictorially we have the following two possibilities:
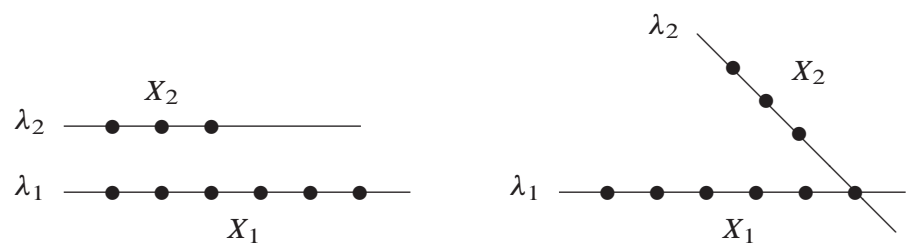

Combining Lemma 2.2 with the fact that $2 X$ contains a subscheme of degree 12 on a line, we get that the difference function of $2 X$ ends in degree exactly 11 . Using 
Bézout it is not hard to check that

$$
\begin{aligned}
& 5 \operatorname{lllh}^{0}\left(d_{2 X}\right)=h^{0}\left(d_{2 X}(1)\right)=h^{0}\left(d_{2 X}(2)\right)=h^{0}\left(d_{2 X}(3)\right)=0 \\
& h^{0}\left(d_{2 X}(4)\right)=1 \\
& h^{0}\left(d_{2 X}(5)\right)=h^{0}\left(d_{2 X_{2}}(3)\right)=h^{0}\left(d_{X_{2}}(2)\right)=3 \\
& h^{0}\left(d_{2 X}(6)\right)=h^{0}\left(d_{2 X_{2}}(4)\right)=h^{0}\left(d_{X_{2}}(3)\right)=7 .
\end{aligned}
$$

This means that the difference function of $2 X$ begins $122344443 \ldots \ldots$ and arguing as in Case 3 gives the result.

Case 5: 1222 2. This case corresponds to nine points on a reduced conic curve. There are three possibilities. If the conic is smooth then the nine points are arbitrary. If the conic consists of two lines then this case takes the form of five points on one line and four points on the other line. Here we can have (i) none of the nine points is the point of intersection of the two lines, or (ii) one of the five points is the point of intersection. All of these cases have been studied in [7], and we omit the details.

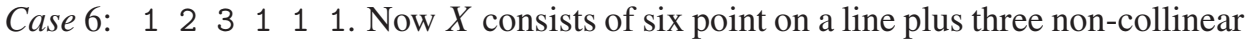
points off the line. It is easy to check, using the same methods, that there is only one possibility for the Hilbert function of $2 X$, independent of whether the line through two of the non-collinear points meets one of the six collinear points or not. We omit the details.

Case 7: 12232 1. By Lemma 2.2, the difference function for $2 X$ ends in degree $\leq 9$ and the entries again add up to 27 . Furthermore, it is not hard to see that $X$ has at most 5 points on a line, and $X$ has at most one set of 5 collinear points.

The first main step in the proof is the following assertion:

Claim 2.6. $h^{0}\left(d_{2 X}(5)\right)=0$.

Note that this implies that $h^{0}\left(\mathcal{l}_{2 X}(t)\right)=0$ for $t \leq 5$. Suppose that there is a curve $F$, of degree 5 containing $2 X$. There are several possibilities. By abuse of notation we will denote by $F$ also a form defining this curve.

- $F$ is reduced. Then $F$ has to contain 9 singular points, which form the points of $X$ (and hence have the difference function $\left.\begin{array}{lllll}1 & 2 & 3 & 2 & 1\end{array}\right)$. This can happen in one of two ways:

- $F$ consists of the union of five lines, and $X$ consists of nine of the resulting ten double points. But from Bézout we note that the 10 double points do not lie on a cubic curve ( since each of the five lines would have to be a component of the cubic), so the ten points have difference function 1234 , and hence $X$ cannot have difference function $\begin{array}{lllll}1 & 2 & 3 & 2 & 1 \text {. }\end{array}$ 
- $F$ consists of the union of three lines and a smooth conic, and $X$ consists of all nine resulting double points. Now the three lines have to be components of any cubic containing $X$, so there is a unique such cubic, and again $X$ does not have difference function $\begin{array}{lllllll}1 & 2 & 3 & 2 & 1\end{array}$.

- $F$ has a double conic. Then all the singular points of $F$ must lie on this conic. But, $X$ does not lie on a conic, so this is impossible.

- $F$ has a double line, i.e. $F=L^{2} G, \operatorname{deg} G=3$. Then $G$ contains at most 3 singular points of $F$. This forces the remaining 6 singular points to be on the line, contradicting the fact that at most 5 points of $X$ can lie on a line.

This concludes the proof of Claim 2.6.

Thanks to Claim 2.6, we now know that the difference function for $2 X$ has the form

$$
\begin{array}{llllll}
1 & 2 & 3 & 4 & 5 & 6
\end{array}
$$

where the last four spaces correspond to entries that are $\geq 0$ and add up to $27-21=6$. Now notice that there is an irreducible flat family of subschemes of degree 9 with

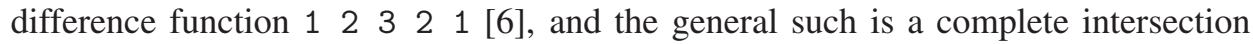
of two cubics. The difference function for the corresponding scheme $2 X$ is easily

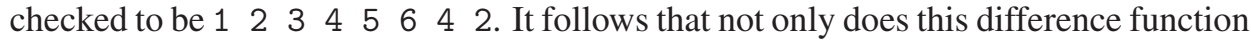
exist, but in fact it corresponds to $\underline{h}^{\max }$. (See also [9, Remark 7.4].) In particular, 1234566 and 12345651 do not occur. The following, then, are the remaining possibilities for the difference function of $2 X$ :

(i) 1223456642

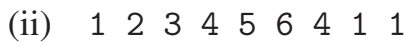

(iii) 1223455633

(iv) $12 \begin{array}{llllllll}1 & 3 & 4 & 5 & 6 & 3 & 2 & 1\end{array}$

(v) $\quad \begin{array}{llllllllll}1 & 2 & 3 & 4 & 5 & 6 & 3 & 1 & 1 & 1\end{array}$

(vi) $1 \begin{array}{lllllllll}1 & 2 & 3 & 4 & 5 & 6 & 2 & 2 & 2\end{array}$

(vii) $\begin{array}{llllllllll}1 & 2 & 3 & 4 & 5 & 6 & 2 & 2 & 1 & 1\end{array}$

For each of these we will either give a specific example (that the reader can verify directly, either by hand or on a computer program) or a proof of non-existence.

(i) $125 \quad 3 \quad 4 \quad 56442$. As we saw above, this occurs when $X$ is the complete intersection of two cubics, and this corresponds to $\underline{h}^{\max }$.

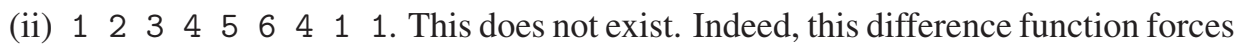
the existence of a line $\lambda$ that contains a subscheme of $2 X$ of degree 9 , which is impossible. (Any such subscheme must have even degree.) 
(iii) $\begin{array}{llllllll}1 & 2 & 3 & 4 & 5 & 6 & 3 & 3\end{array}$. This does not exist in our context. Note that it does exist when $X$ has difference function 1233 , as we will verify below. To see that this does not exist, note that by Theorem 2.3, the 33 at the end forces the existence of a cubic curve $C$ that cuts out from $2 X$ a subscheme $W$ of degree 21 with difference function $\begin{array}{llllllll}1 & 2 & 3 & 3 & 3 & 3 & 3 & 3\end{array}$. Observe that if $P$ is a point of $X$ which is a smooth point of $C$, then $C$ cuts out a non-reduced point of degree 2 at $P$. If $P$ is a point of $X$ which is a singular point of $C$, then $C$ contains the fat point $2 P$ (which has degree 3 ). Note also that our $h$-vector does not permit the existence of a subscheme of degree more than 8 on a line.

Suppose first that $C$ is reduced. Since we only have the nine points of $X$ to work with, it is not hard to check, using the above observation, that the only way that $C$ can cut out from $2 X$ a subscheme of degree 21 is if $X$ has the following configuration:

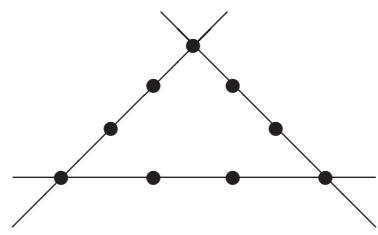

But this uses all nine points, and its support lies on a unique cubic, contradicting the fact that $X$ has difference function $\begin{array}{lllllll}1 & 2 & 3 & 2 & 1 \text {. This configuration provides }\end{array}$ one of the correct difference functions for $\begin{array}{llll}2 & 3 & 3 & \text { below. }\end{array}$

Now suppose that $C$ is not reduced. Without loss of generality, $C$ has a double line. The difference function for $X$ would, in principle, allow five points of $X$ to lie on a line, but because the hypothetical difference function for the subscheme $W$ ends in degree 7 , in fact at most four points of $X$ can lie on a line. So the double line contains at most four fat points of $2 X$, which have degree 12 . In order for $C$ to cut out a subscheme of degree 21 , then, we must have a reduced line that cuts out an additional subscheme of degree at least 9 . This forces at least five points of $X$ to be collinear, which again is impossible.

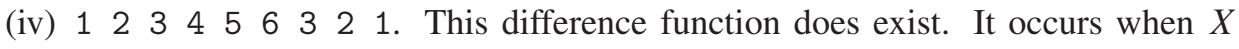
is the union of one point and the complete intersection of a conic and a general quartic curve.

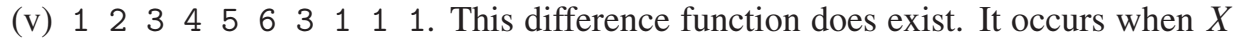
is the union of five general points on a line, three general points on a second line, and one additional general point off both lines.

(vi) $\begin{array}{lllllllll}1 & 2 & 3 & 4 & 5 & 6 & 2 & 2 & 2\end{array}$. This difference function does not exist. Indeed, suppose that it did exist. Because of the 22 2, there must be a curve $C$ of degree 2 that cuts out on $2 X$ a subscheme $W$ of degree 17 having difference function $\begin{array}{lllllllll}1 & 2 & 2 & 2 & 2 & 2 & 2 & 2 & 2 .\end{array}$ 
First note that $X$ cannot contain five points on a line (and hence a subscheme of $W$ of degree at least 10) since the hypothetical difference function ends in degree 8. Now consider cases.

(a) $C$ is smooth: then it cannot cut out a subscheme of odd degree.

(b) $C$ is reduced and reducible: then we cannot obtain the desired subscheme $W$ of degree 17 unless $X$ contains 5 points on a line, in which case $W$ contains a subscheme of degree at least 10 on that line.

(c) $C$ non-reduced: then we cannot have a subscheme of degree 17 supported on that line.

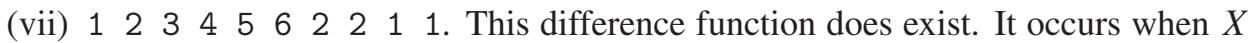
has the following configuration:

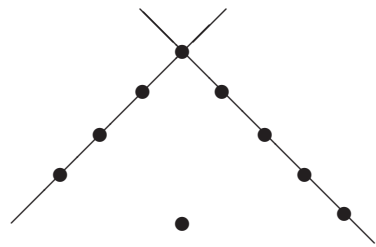

Case 8: 1223 3. This is the difference function for a general set of nine points in $\mathbb{P}^{2}$. We know (from [12], for example) that the "generic" difference function for nine general double points is $\begin{array}{lllllll}2 & 3 & 4 & 5 & 6 & 6\end{array}$. Hence this occurs and corresponds to the maximum possible Hilbert function. Clearly all other possibilities will end in degree $\geq 7$. On the other hand, Lemma 2.2 guarantees that all other examples end in degree $\leq 7$. Note that again, $X$ can have at most four points on a line.

Claim 8.1. $h^{0}\left(d_{2 X}(5)\right) \leq 1$.

Notice that as a consequence of this claim we also obtain $h^{0}\left(d_{2 X}(4)\right)=0$. Keeping in mind that it is possible that $h^{0}\left(\mathscr{l}_{2 X}(5)\right)=0$ (e.g. the generic case), we will assume that $h^{0}\left(\mathcal{l}_{2 X}(5)\right) \neq 0$ and deduce that then it must be $=1$. So let $C$ be a curve of degree 5 containing the scheme $2 X$. As before (Claim 2.6) there are a few possibilities.

- If $C$ is reduced then since it must have nine double points, it must consist of either the union of five lines, no three through a point, or the union of three lines and a smooth conic, with no three components meeting in a point. By Bézout, each component of $C$ is then a fixed component of the linear system $\left|\left(I_{2 X}\right)_{5}\right|$, so the claim follows.

- If $C$ contains a double line then at most four (fat) points of $2 X$ lie on this line, so we must have a cubic curve that contains the remaining five double points. Consider the support, $X_{1}$, of these five double points. The points of $X_{1}$ are not collinear, and they do not have four collinear points since $X$ lies on only one 
cubic. With these restrictions, clearly there is no cubic curve double at such a set of five points.

- If $C$ contains a double conic (smooth or not), this conic contains at most seven points of $X$, because of the Hilbert function of $X$. Hence $C$ must have a line that contains two double points, which is impossible.

This concludes the proof of Claim 8.1.

It follows that the possibilities for the difference function of $2 X$ are the following:

(i) 122345566

(ii) 1223455651

(iii) $122 \quad 3 \quad 4 \quad 5 \quad 6 \quad 42$

(iv) 122345633

(v) 12345552

(vi) 1223455554

As before, we examine these each in turn.

(i) 12234456 . We have seen that this occurs generically.

(ii) 122345665 . This exists, for instance from the following configuration:

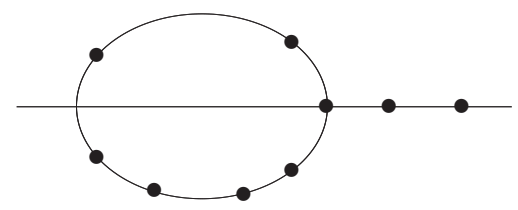

(That is, seven points on a conic, three points on a line, with one point in common.)

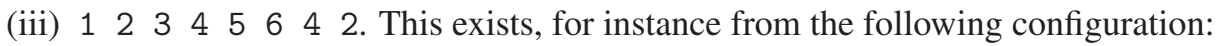

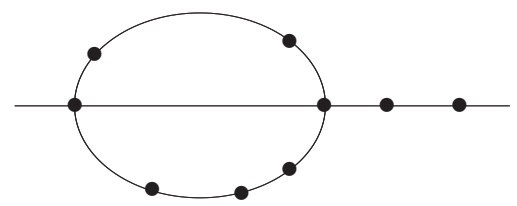


(iv) $\begin{array}{llllllll}1 & 2 & 3 & 4 & 5 & 6 & 3 & 3\end{array}$. This exists, for instance from the configuration mentioned earlier:

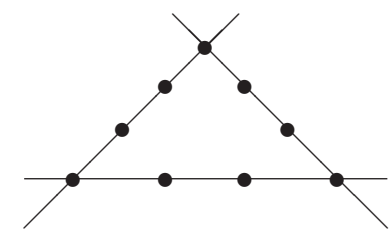

(v) 1223445555 2. We claim that this does not exist. The key is that such a double point scheme, $2 X$, would have to lie on a unique quintic curve, say $C$. To see that this is impossible, the argument is very similar to that of Claim 2.6, but with a small difference. One checks as before that $C$ must consist either of five lines or the union of three lines and a conic, and in both cases we must have that no three components share a common point. In the first case, $X$ consists of nine of the ten double points of $C$ (it does not matter which nine), and in the second case $X$ consists of all nine double points of $C$. But in both of these cases one can check geometrically or on a computer that $h^{0}\left(\mathcal{l}_{2 X}(6)\right)=4$, while the hypothetical difference function would require this dimension to be 3 .

(vi) $122 \quad 3 \quad 4 \quad 5 \quad 5 \quad 4 \quad 3$. This exists, and can be achieved by the configuration mentioned above: it is supported on nine of the ten intersection points of five general lines in $\mathbb{P}^{2}$.

\section{Approach I: Points on Cubics}

For this section we will always let $C \subset \mathbb{P}^{2}$ be an irreducible cubic curve defined by a polynomial $F$ of degree 3 . Let $X$ be a reduced set of $n=3 t+\delta$ points on $C$, where $0 \leq \delta \leq 2$. Let $Z=2 X$ be the double point scheme in $\mathbb{P}^{2}$ supported on $X$. The object of this section is to describe the possible Hilbert functions of $X$ and of the corresponding $Z$. In some instances we assume that $t$ is "big enough" (with mild bounds), and in one instance (Theorem 3.4 (ii)) we assume that the points are not too special and that $C$ is smooth.

Proposition 3.1. Assume that $\delta=0, t \geq 3$, and the Hilbert function of $X$ has first difference

$$
\begin{array}{c|cccccccc}
\operatorname{deg} & 0 & 1 & 2 & \ldots & t-1 & t & t+1 & t+2 \\
\hline \Delta h_{X} & 1 & 2 & 3 & \ldots & 3 & 2 & 1 & 0
\end{array}
$$

(where the values between 2 and $t-1$, if any, are all 3). Then $X$ is a complete intersection with ideal $(F, G)$, where $\operatorname{deg} F=3$ and $\operatorname{deg} G=t$. Furthermore, if $C$ 
is singular then the singular point is not a point of $X$. Assume that $t>3$, so that $t+3>6$ and $2 t>t+3$. Then we have the first difference of the Hilbert function of $Z$ is

$$
\begin{aligned}
& (t=3) \quad 123456420 ; \\
& (t=4) \quad 12345665310 ; \\
& (t=5) \quad 1234566654210 ; \\
& (t \geq 6)
\end{aligned}
$$

\begin{tabular}{l|ccccccccccc}
$\operatorname{deg}$ & 0 & 1 & 2 & 3 & 4 & 5 & 6 & $\ldots$ & $t+2$ & $t+3$ \\
\hline$\Delta h_{Z}$ & 1 & 2 & 3 & 4 & 5 & 6 & 6 & $\ldots$ & 6 & 5 \\
$\operatorname{deg}$ & $t+4$ & $t+5$ & $\ldots$ & $2 t-1$ & $2 t$ & $2 t+1$ & $2 t+2$ \\
\hline$\Delta h_{Z}$ & 4 & 3 & $\ldots$ & 3 & 2 & 1 & 0
\end{tabular}

Proof. We first show that $X$ must be a complete intersection. From the Hilbert difference function (3.1), it is clear that $F$ is a factor of every form in $I_{X}$ up to degree $t-1$, and that in fact it generates the ideal up to this point. In degree $t$ there is exactly one new form, $G$, in the ideal, and since $F$ is irreducible, $F$ and $G$ have no common factor. But $(F, G)$ is a saturated ideal that is contained in $I_{X}$ and defines a zero-dimensional scheme of the same degree as $X$, hence $I_{X}=(F, G)$.

Since $X$ is a complete intersection, if $C$ is singular and $P \in X$ is the singular point of $C$, then $X$ must be non-reduced at $P$, contradicting our assumption.

Now, it is a simple (and standard) argument that $I_{Z}=\left(F^{2}, F G, G^{2}\right)$, and one can verify the claimed Hilbert function of $R / I_{Z}$, for instance by using the fact that $(F, G)$ is directly linked to the ideal of $Z$ by the complete intersection $\left(F^{2}, G^{2}\right)$, and using the formula for the behavior of Hilbert functions under linkage [5] (see also [15]). We omit the details. See also [3, pages 176, 177], and [2, Remark 4.9].

Because the form $F$ of least degree is irreducible, the Hilbert function of $X$ has first difference that is strictly decreasing from the first degree where it has value $<3$ until it reaches 0 . Having proved Proposition 3.1, we can now assume without loss of generality that the Hilbert function of $X$ has first difference

\begin{tabular}{c|cccccccc}
$\operatorname{deg}$ & 0 & 1 & 2 & 3 & $\ldots$ & $t$ & $t+1$ & $t+2$ \\
\hline$\Delta h_{X}$ & 1 & 2 & 3 & 3 & $\ldots$ & 3 & $\delta$ & 0
\end{tabular}

where $0 \leq \delta \leq 2$

Theorem 3.4. Assume that either $C$ is smooth, or else that no point of $X$ is the singular point of $C$. Assume further that $t>5-\delta$. Then the Hilbert difference function of the double point scheme $Z$ supported on $X$ is

\begin{tabular}{c|ccccccccccccc}
$\operatorname{deg}$ & 0 & 1 & 2 & 3 & 4 & 5 & $\ldots$ & $t+3$ & $t+4$ & $t+5$ & $\ldots$ & $2 t+\delta-1$ & $2 t+\delta$ \\
\hline$\Delta h_{Z}$ & 1 & 2 & 3 & 4 & 5 & 6 & $\ldots$ & 6 & $3+\delta$ & 3 & $\ldots$ & 3 & $? ?$
\end{tabular}


For the behavior in degree $\geq 2 t+\delta$, we have the following conclusions.

(i) If $\delta=1$ or $\delta=2$ then $\Delta h_{Z}(2 t+\delta)=3-\delta$ and $\Delta h_{Z}(k)=0$ for $k>2 t+\delta$.

(ii) If $\delta=0$, there are two possible Hilbert functions, these being determined by

(a) $\Delta h_{Z}(2 t)=3$ and $\Delta h_{Z}(k)=0$ for $k>2 t$, and

(b) $\Delta h_{Z}(2 t)=2, \Delta h_{Z}(2 t+1)=1, \Delta h_{Z}(2 t+2)=0$.

Moreover, if the points $p_{i}$ are sufficiently general and $C$ is smooth, then the Hilbert function is given by the first of these two.

Proof. A complete analysis of all cases with $\delta=0$, where $C$ is a reduced cubic and the points $p_{i}$ either are arbitrary smooth points of $C$ or they are completely arbitrary and $C$ is also irreducible, is given in the next section using the Second Way. The interested reader can complete the current proof to those cases using the techniques of this section, as a further comparison of the methods.

First note that the condition $t>5-\delta$ implies $2 t+\delta>t+5$. We proceed via a number of claims.

Claim 1. For $\ell<2 t+\delta,\left(I_{Z}\right)_{\ell}$ has the cubic form $F$ as a common factor (i.e. $C$ is part of the base locus).

Suppose that $G \in\left(I_{Z}\right)_{\ell}$ does not have $F$ as a factor. Then at each point of $X$, the intersection multiplicity of $F$ and $G$ is at least 2 since $G$ is double at each point. Hence by Bézout's theorem, $3 \ell \geq 2 n=2(3 t+\delta)=6 t+2 \delta$. Hence $\ell \geq 2 t+\frac{2}{3} \delta$, and the claim follows.

Claim 2. For $\ell \leq t+3,\left(I_{Z}\right)_{\ell}$ has $F^{2}$ as a common factor.

By Claim 1, since $F$ is not double at any point of $X$, for $\ell<2 t+\delta$ we have an isomorphism

$$
\left(I_{X}\right)_{\ell-3} \cong\left(I_{Z}\right)_{\ell}
$$

where the isomorphism is given by multiplication by $F$. But from (3.2), we see that $F$ is a common factor for $\left(I_{X}\right)_{k}$ for all $k \leq t$. Hence $\left(I_{Z}\right)_{\ell}$ has $F^{2}$ as a factor whenever $\ell-3 \leq t$, as claimed.

This verifies the claimed first difference of the Hilbert function up to degree $t+3$. Note that the Hilbert function, in degree $t+3$, has value equal to

$$
1+2+3+4+5+6 \cdot[(t+3)-4]=6 t+9 \text {. }
$$


We now compute the value in degree $t+4<2 t$. Using the isomorphism (3.3), we have

$$
\begin{aligned}
h_{Z}(t+4) & =\left(\begin{array}{c}
t+6 \\
2
\end{array}\right)-h^{0}\left(d_{Z}(t+4)\right) \\
& =\left(\begin{array}{c}
t+6 \\
2
\end{array}\right)-h^{0}\left(d_{X}(t+1)\right) \\
& =\left(\begin{array}{c}
t+6 \\
2
\end{array}\right)-\left[\left(\begin{array}{c}
t+3 \\
2
\end{array}\right)-h_{X}(t+1)\right] \\
& =\left(\begin{array}{c}
t+6 \\
2
\end{array}\right)-\left[\left(\begin{array}{c}
t+3 \\
2
\end{array}\right)-(3 t+\delta)\right] \\
& =6 t+12+\delta .
\end{aligned}
$$

Then we easily see that $\Delta h_{Z}(t+4)=3+\delta$ as claimed.

Next we compute the value in degree $t+5$. We have $2 t+\delta>t+5$, so we can use Claim 1. Then a similar computation gives

$$
h_{Z}(t+5)=6 t+15+\delta .
$$

From this we immediately confirm $\Delta h_{Z}(t+5)=3$.

Since $F$ is a common factor in all components $<2 t+\delta$, and since $\Delta h_{Z}$ takes the value 3 already in degree $t+5$, it repeats this value until $F$ is no longer a common factor. In particular, it takes the value 3 up to degree $2 t+\delta-1$.

We now have to see what happens past degree $2 t+\delta-1$. Note that using our above calculations, it follows that

$$
\begin{aligned}
h_{Z}(2 t+\delta-1) & =6 t+12+\delta+3[2 t+\delta-1-(t+4)] \\
& =3(3 t+\delta)-3+\delta .
\end{aligned}
$$

Since $\operatorname{deg} Z=3(3 t+\delta)$, we have reached the multiplicity minus $(3-\delta)$. We consider these cases separately. When $\delta=1$ or $\delta=2$, we are adding only 2 or 1 , respectively, and since the first difference of the Hilbert function cannot be flat at this point, $\Delta h_{Z}$ must be as claimed in (a). This completes (a). Since the sum of the values of $\Delta h_{Z}$ up to degree $2 t-1$ is $9 t-3$, this observation that $\Delta h_{Z}$ cannot be flat at this point also proves that the possibilities listed in (b) are the only ones possible.

If $\delta=0$, though, $\Delta h_{Z}$ can either end $\ldots 3,3,0$ or $\ldots 3,2,1$. We now consider these two possibilities. The former means that also in degree $2 t+\delta=2 t$, all forms in $I_{Z}$ have $F$ as a factor. The latter means that there is a form, $G$, of degree $2 t+\delta=2 t$ in $I_{Z}$ that does not have $F$ as a factor, and hence $(F, G)$ is a regular sequence (since $F$ is irreducible). 
Suppose that the latter holds. Note that the complete intersection defined by $(F, G)$ has degree $3 \cdot 2 t=6 t=2 n$. As in Claim $1, G$ cuts out on $C$ a divisor of degree at least $2 n$, so in fact $G$ cuts out exactly the divisor $2 X_{C}$ on $C$ (where by $2 X_{C}$ we mean the subscheme $2 X \cap C$, which is a divisor on $C$ ). So $X$ itself is not a complete intersection (since it has the Hilbert difference function given by (3.2)), but the divisor $2 X_{C}$ (as a subscheme of $\mathbb{P}^{2}$ ) is a complete intersection, namely of type $(3,2 t)$. Note that $2 X_{C}$, which is curvilinear, is not the same as $Z$.

Now suppose that $C$ is smooth. We know that then two effective divisors of the same degree are linearly equivalent if and only if they have the same sum in the group of $C$. The condition described in the previous paragraph implies that the divisor $X_{C}-n Q$, where $Q$ is a point of inflection for $C$, is a 2-torsion element in the Picard group of $C$ but is not zero. Since there are at most three 2-torsion elements in the Picard group of $C$, for general choices we have a contradiction, and so such a $G$ cannot exist (in general), and we have proved the assertion about the general choice of the points.

Finally, we show that the Hilbert difference function (b) of (ii) also occurs. We begin with four general lines, $\lambda_{1}, \lambda_{2}, \lambda_{3}, \lambda_{4} \subset \mathbb{P}^{2}$ and let $P_{1}, P_{2}, P_{3}, P_{4}, P_{5}, P_{6}$ be the six points of pairwise intersection of these lines. Let $G_{1}$ be the form defining the union of these four lines. Let $X_{1}=\bigcup_{1 \leq i \leq 6} P_{i}$. Notice that $X_{1}$ does not lie on any conic, since by Bézout any conic containing $X_{1}$ has to contain all four lines $\lambda_{1}, \ldots, \lambda_{4}$, hence must have $G_{1}$ as a factor. Thus the Hilbert function of $X_{1}$ has first difference $(1,2,3)$, and $X_{1}$ is not a complete intersection.

Let $C$ be a general cubic curve containing $X_{1}$ (hence $C$ is smooth), and let $F$ be the defining polynomial of $C$. Notice that the degree of the complete intersection of $F$ and $G_{1}$ is 12 , and this complete intersection is at least double at each $P_{i}$, so in fact it is exactly double at each $P_{i}$. In particular, there is no additional multiplicity at any of the $P_{i}$ coming from tangency. As a divisor on $C$, note that $X_{1}$ is not cut out by any conic, since it is not a complete intersection. However, the divisor $2 X_{1}$ is cut out by a quartic, namely $G_{1}$.

Now let $X$ be the union of $X_{1}$ with a general hypersurface section, $W_{1}$, of $C$ cut out by a curve of degree $t-2$. Note that $W_{1}$ is a complete intersection defined by $(F, H)$ for some form $H$ of degree $t-2$. We first claim that $X$ is not a complete intersection. Indeed, suppose that $X$ were a complete intersection defined by $\left(F, H^{\prime}\right)$ for some $H^{\prime}$ of degree $t$. Then $I_{X}$ links $W_{1}$ to $X_{1}$. But $W_{1}$ and $X$ are both complete intersections sharing a generator, so by liaison theory the residual is also a complete intersection. But we have seen that $X_{1}$ is not a complete intersection. Contradiction. In particular, $\Delta h_{X}$ is given by (3.2).

Now let $Z$ be the fat point scheme supported on $X$, and consider the form $G_{1} H^{2}$. This has degree $2 t$, and cuts out the divisor $2 X$ on $C$. Even more, $G_{1} H^{2}$ is an element of $I_{Z}$ in degree $2 t$ that does not have $F$ as a factor. As we saw above, this gives the values $\Delta h_{Z}(2 t)=2$ and $\Delta h_{Z}(2 t+1)=1$ as desired. This completes the proof of Theorem 3.4. 
Now we wish to explore the possibilities when $C$ is singular and one point, $P$, of $X$ is the singular point of $C$. The arguments are very similar, and we will primarily highlight the differences. The main observation is that $C$ is already double at $P$ so we have to focus on the remaining $n-1$ points.

Lemma 3.6. Assume that $C$ is singular, that $P \in X \subset C$ is the singular point of $C$, and that $n \geq 5$. Then $X$ is not a complete intersection.

Proof. More precisely, we will show that if $P \in X \subset C$ with $X$ a complete intersection, and if $P$ is the singular point of $C$, then $X$ has one of the following types: $C I(1,1), C I(1,2), C I(2,2)$.

First note that if $X$ is a complete intersection defined by forms $(F, G)$, where $F$ is the defining polynomial for $C$, then $X$ has multiplicity $\geq 2$ at $P$, so $X$ is not reduced. Hence we have to determine all the possibilities for reduced complete intersections on $C$ that do not use $F$ as a minimal generator. The listed possibilities are clear: one point, two points, four points, and these all exist even including $P$ as one of the points. Using the irreducibility of $F$, it is not hard to show that these are the only possibilities, and we omit the details.

Theorem 3.7. Assume that $C$ is an irreducible singular cubic with singular point $P$, and assume that $P \in X$, where $X$ is a reduced set of $3 t+\delta$ points of $C$, with $0 \leq \delta \leq 2$. Assume further that $t>3$. Then the Hilbert difference function of the double point scheme $Z$ supported on $X$ is as follows.

(i) If $\delta=0$ then

\begin{tabular}{|c|c|c|c|c|c|c|c|c|c|c|c|}
\hline deg & 0 & 23 & 4 & . & $t+2$ & $t+3$ & $t+4$ & $t+5$ & $2 t$ & $2 t+1$ & $2 t+2$ \\
\hline$\Delta h_{Z}$ & 12 & 34 & 5 & & 6 & 5 & 3 & 3 & 3 & 1 & 0 \\
\hline
\end{tabular}

(ii) If $\delta=1$ then either

\begin{tabular}{c|ccccccccccccccc}
$\operatorname{deg}$ & 0 & 1 & 2 & 3 & 4 & 5 & $\ldots$ & $t+2$ & $t+3$ & $t+4$ & $t+5$ & $\ldots$ & $2 t$ & $2 t+1$ & $2 t+2$ \\
\hline$\Delta h_{Z}$ & 1 & 2 & 3 & 4 & 5 & 6 & $\ldots$ & 6 & 6 & 3 & 3 & $\ldots$ & 3 & 3 & 0
\end{tabular}

or

\begin{tabular}{c|ccccccccccccccc}
$\operatorname{deg}$ & 0 & 1 & 2 & 3 & 4 & 5 & $\ldots$ & $t+2$ & $t+3$ & $t+4$ & $t+5$ & $\ldots$ & $2 t$ & $2 t+1$ & $2 t+2$ \\
\hline$\Delta h_{Z}$ & 1 & 2 & 3 & 4 & 5 & 6 & $\ldots$ & 6 & 5 & 4 & 3 & $\ldots$ & 3 & 3 & 0
\end{tabular}

(iii) If $\delta=2$ then

$$
\begin{array}{c|cccccccccccccccc}
\operatorname{deg} & 0 & 1 & 2 & 3 & 4 & 5 & \ldots & t+2 & t+3 & t+4 & t+5 & \ldots & 2 t & 2 t+1 & 2 t+2 & 2 t+3 \\
\hline \Delta h_{Z} & 1 & 2 & 3 & 4 & 5 & 6 & \ldots & 6 & 6 & 4 & 3 & \ldots & 3 & 3 & 2 & 0
\end{array}
$$


Proof. The bound $t>3$ is simply to ensure that in each case, some value of the Hilbert difference function $\Delta h_{Z}$ takes the value 3. For instance, in the case $\delta=0$, we have $2 t>t+3$. As a consequence of Lemma 3.6, when $n=3 t+\delta \geq 5$ the Hilbert function of $X$ must have first difference

$$
\begin{array}{c|cccccccc}
\operatorname{deg} & 0 & 1 & 2 & 3 & \ldots & t & t+1 & t+2 \\
\hline \Delta h_{X} & 1 & 2 & 3 & 3 & \ldots & 3 & \delta & 0
\end{array}
$$

In analogy with Theorem 3.4, we first have

Claim 1. Assume that

$$
\ell \leq \begin{cases}2 t & \text { if } \delta=0 \\ 2 t+1 & \text { if } \delta=1,2\end{cases}
$$

Then $F$ is a common factor of $\left(I_{Z}\right)_{\ell}$.

The proof is the same as that of Claim 1 in Theorem 3.4, except that the intersection multiplicity of $F$ and $G$ at $P$ is now at least 4 .

Claim 2. For $\ell \leq t+2,\left(I_{Z}\right)_{\ell}$ has $F^{2}$ as a common factor. Furthermore,

- If $\delta=0$ then $F^{2}$ is not a common factor of $\left(I_{Z}\right)_{t+3}$.

- If $\delta=2$ then $F^{2}$ is a common factor of $\left(I_{Z}\right)_{t+3}$.

- If $\delta=1$ then $F^{2}$ may or may not be a common factor of $\left(I_{Z}\right)_{t+3}$ (examples exist for either option).

The proof of Claim 2 hinges on the possible Hilbert functions for $X-\{P\}$. In particular, we show that $\left(I_{X-\{P\}}\right)_{t-1}$ always has $F$ as a common factor, and the differences in the three cases rest with the possibilities for $\left(I_{X-\{P\}}\right)_{t}$, which we get by comparing to those for $I_{X}$, obtained using Lemma 3.6.

- If $\delta=0$ then $X$ has Hilbert function with first difference

$$
\begin{array}{c|ccccccc}
\operatorname{deg} & 0 & 1 & 2 & 3 & \ldots & t & t+1 \\
\hline \Delta h_{X} & 1 & 2 & 3 & 3 & \ldots & 3 & 0
\end{array}
$$

so clearly the only possibility for $\Delta h_{X-\{P\}}$ is

\begin{tabular}{l|cccccccc}
$\operatorname{deg}$ & 0 & 1 & 2 & 3 & $\ldots$ & $t-1$ & $t$ & $t+1$ \\
\hline$\Delta h_{X-\{P\}}$ & 1 & 2 & 3 & 3 & $\ldots$ & 3 & 2 & 0
\end{tabular}

Hence there is a form $G$ of degree $t$ vanishing on $X-\{P\}$ but not containing $F$ as a factor, so $F G \in\left(I_{Z}\right)_{t+3}$ does not have $F^{2}$ as a factor. 
- If $\delta=2$ then $X$ has Hilbert function with first difference

$$
\begin{array}{l|ccccccccc}
\operatorname{deg} & 0 & 1 & 2 & 3 & \ldots & t-1 & t & t+1 & t+2 \\
\hline \Delta h_{X-\{P\}} & 1 & 2 & 3 & 3 & \ldots & 3 & 3 & 2 & 0
\end{array}
$$

so $\Delta h_{X-\{P\}}$ is

\begin{tabular}{l|ccccccccc}
$\operatorname{deg}$ & 0 & 1 & 2 & 3 & $\ldots$ & $t-1$ & $t$ & $t+1$ & $t+2$ \\
\hline$\Delta h_{X-\{P\}}$ & 1 & 2 & 3 & 3 & $\ldots$ & 3 & 3 & 1 & 0
\end{tabular}

We know that $\left(I_{Z}\right)_{\ell} \cong\left(I_{X-\{P\}}\right)_{\ell-3}$ for $\ell$ satisfying the bounds of Claim 1 , and as a result of the above observations we know when $\left(I_{X-\{P\}}\right)_{\ell-3}$ is forced to have $F$ as a common factor, so the claim follows.

If $\delta=1$ then $X$ has Hilbert function with first difference

\begin{tabular}{c|cccccccc}
$\operatorname{deg}$ & 0 & 1 & 2 & 3 & $\ldots$ & $t$ & $t+1$ & $t+2$ \\
\hline$\Delta h_{X}$ & 1 & 2 & 3 & 3 & $\ldots$ & 3 & 1 & 0
\end{tabular}

so $\Delta h_{X-\{P\}}$ is either

\begin{tabular}{l|cccccccc}
$\operatorname{deg}$ & 0 & 1 & 2 & 3 & $\ldots$ & $t-1$ & $t$ & $t+1$ \\
\hline$\Delta h_{X-\{P\}}$ & 1 & 2 & 3 & 3 & $\ldots$ & 3 & 3 & 0
\end{tabular}

or

\begin{tabular}{l|ccccccccc}
$\operatorname{deg}$ & 0 & 1 & 2 & 3 & $\ldots$ & $t-1$ & $t$ & $t+1$ & $t+2$ \\
\hline$\Delta h_{X-\{P\}}$ & 1 & 2 & 3 & 3 & $\ldots$ & 3 & 2 & 1 & 0
\end{tabular}

Since we have removed $P$, the remaining points could be a complete intersection, so $F^{2}$ is a common factor of $\left(I_{Z}\right)_{t+3}$ if and only if the points of $X-\{P\}$ are not a complete intersection of a curve of degree $t$ with $F$. This completes the proof of Claim 2.

The rest of the proof is very similar to that of Theorem 3.4 and we omit the details.

\section{Approach II: Points on Cubics}

Let $Z=m_{1} p_{1}+\cdots+m_{n} p_{n} \subset \mathbb{P}^{2}$, where the points $p_{1}, \ldots, p_{n}$ are distinct and arbitrary. When $n<9$, a complete determination of $h_{Z}$ is given in [7], but the case of $n \geq 9$ remains of interest. Giving a complete determination of $h_{Z}$ for all $n \geq 9$ arbitrary distinct points $p_{1}, \ldots, p_{n}$ would involve solving some extremely hard open problems. For example, it is even an open problem to determine $h_{Z}$ for $n>9$ when the points $p_{1}, \ldots, p_{n}$ are general and $m_{1}=\cdots=m_{n}$. So here, as in Section 3, we consider the case of $n \geq 9$ points $p_{i}$ in special cases. These cases include those 
considered in Section 3. We recover and in some cases extend the results of Section 3, but the methods we use here are different. To start, let $p_{1}, \ldots, p_{n}$ be $n \geq 9$ distinct points on a reduced plane cubic $C$. If $C$ is not irreducible, we assume further that all the points are smooth points of $C$. If $D$ is a component of $C$, let $n_{D}$ be the number of these points on $D$. We will say that the points are evenly distributed if $n_{D}=$ $n(\operatorname{deg}(D)) / 3$ for every reduced irreducible component $D$ of $C$. Note that for $n$ points to be evenly distributed, it is necessary either that 3 divide $n$ or that $C$ be irreducible.

We will use some facts about surfaces obtained by blowing up points in the plane, in particular we'll make use of the intersection form on such surfaces, which we now briefly recall. Given distinct points $p_{1}, \ldots, p_{n} \in \mathbb{P}^{2}$, let $\pi: Y \rightarrow \mathbf{P}^{2}$ be the morphism obtained by blowing up the points $p_{i}$. The divisor class group $\mathrm{Cl}(Y)$ of divisors modulo linear equivalence is a free Abelian group with basis $[L],\left[E_{1}\right], \ldots,\left[E_{n}\right]$, where $L$ is the pullback to $Y$ of a general line, and $E_{i}=\pi^{-1}\left(p_{i}\right)$. There is a bilinear form, called the intersection form, defined on the group of divisors, which descends to $\mathrm{Cl}(Y)$. It is uniquely determined by the fact that $L, E_{1}, \cdots, E_{n}$ are orthogonal with respect to the intersection form, with $L \cdot L=L^{2}=1$ and $E_{i}^{2}=-1$ for $i=1, \ldots, n$. For two distinct, reduced, irreducible curves $C_{1}$ and $C_{2}$ on $Y, C_{1} \cdot C_{2}$ is just the number of points of intersection of the two curves, counted with multiplicity. We recall that a divisor $F$ is nef if $F \cdot C \geq 0$ for every effective divisor $C$. A useful criterion for nefness is that if $F$ is an effective divisor such that $F \cdot C \geq 0$ for every component $C$ of $F$, then $F$ is nef.

In preparation for stating Theorem 4.2, our main result in this section, we set some additional notation. Let $Z=m\left(p_{1}+\cdots+p_{n}\right)$. When $t$ satisfies $3 t=m n$, the value of $h_{Z}(t)$ is influenced by torsion in the group $\operatorname{Pic}(C)$. Our formula for $h_{Z}$ as given in Theorem 4.2 accounts for this influence via an integer-valued function we will denote by $s$. In fact, $s$ depends on the points $p_{i}$, on $m$ and on $t$, but for a fixed set of points $p_{i}$ it is convenient to mostly suppress the dependence on the points and denote $s$ as $s(t, n, m)$, where the parameter $n$ is a reminder of the dependence on the $n$ points. To define $s(t, n, m)$, let $L$ be a general line in the plane and fix evenly distributed smooth points $p_{1}, \ldots, p_{n}$ of a reduced cubic $C$. Since Theorem 4.2 applies only for $n \geq 9$ and we need $s(t, n, m)$ only when $t \geq n m / 3$, we define $s(t, n, m)$ only for $n \geq 9$ when $t \geq n m / 3$ :

(i) If $t>n m / 3$, we set $s(t, n, m)=0$.

(ii) If $n=9$ and $t=3 m$, let $\lambda$ be the order (possibly infinite) of $\mathcal{O}_{C}(3 L) \otimes$ $\mathcal{O}_{C}\left(-p_{1}-\cdots-p_{9}\right)$ in $\operatorname{Pic}(C)$. We then set $s(t, n, m)=\lfloor m / \lambda\rfloor$.

(iii) If $n>9$ and $t=n m / 3$, we set $s(t, n, m)=1$ if $\mathcal{O}_{C}(t L) \otimes \mathcal{O}_{C}\left(-m p_{1}-\cdots-\right.$ $\left.m p_{n}\right)=\mathcal{O}_{C}$ in $\operatorname{Pic}(C)$, and we set $s(t, n, m)=0$ otherwise.

The value of $s(t, n, m)$ depends on whether $\mathcal{O}_{C}(t L) \otimes \mathcal{O}_{C}\left(-m p_{1}-\cdots-m p_{n}\right)$ is trivial. Note that triviality of this line bundle is equivalent to the divisor $m p_{1}+$ $\cdots+m p_{n}$ on $C$ being the intersection of $C$ with a curve $H$, necessarily of degree $t=m n / 3$. Of course it can happen that $\mathcal{O}_{C}(t L) \otimes \mathcal{O}_{C}\left(-p_{1}-\cdots-p_{n}\right)$ is non-trivial 
even though $\mathcal{O}_{C}(t m L) \otimes \mathcal{O}_{C}\left(-m p_{1}-\cdots-m p_{n}\right)$ is trivial. For example, if $p_{1}, p_{2}$ and $p_{3}$ are flexes on $C$ but not collinear, then $\mathcal{O}_{C}(L) \otimes \mathcal{O}_{C}\left(-p_{1}-p_{2}-p_{3}\right)$ is not trivial, but $\mathcal{O}_{C}(3 L) \otimes \mathcal{O}_{C}\left(-3 p_{1}-3 p_{2}-3 p_{3}\right)$ is trivial, and $H$ in this case is the union of the lines tangent to $C$ at the points $p_{1}, p_{2}$ and $p_{3}$. When $C$ is a smooth cubic curve, triviality of $\mathcal{O}_{C}(t L) \otimes \mathcal{O}_{C}\left(-m p_{1}-\cdots-m p_{n}\right)$ is equivalent to the sum $m p_{1}+\cdots+m p_{n}$ being trivial in the group law on the cubic (with respect to a flex being taken as the identity element). (The divisor $X_{1}$ given in the proof of part (ii) of Theorem 3.4 gives another example, and shows that this issue arose also with the first approach.)

Remark 4.1. When $n=9$, the values of $\lambda$ that can occur depend on the torsion in $\operatorname{Pic}(C)$, and this depends on $C$ and on the characteristic of the ground field; see Remark 4.4. Thus knowing something about $C$ tells us something about what Hilbert functions can occur for points on $C$, but the Hilbert functions themselves depend only on $\lambda$, and already for a smooth irreducible non-supersingular cubic $C$, there is torsion of all orders.

Theorem 4.2. Let $X=p_{1}+\cdots+p_{n}$ be a set of $n \geq 9$ evenly distributed smooth points on a reduced plane cubic $C$. Let $Z=m X$. The value $h_{Z}(t)=\operatorname{dim}\left(k\left[\mathbf{P}^{2}\right] /(I(Z))\right)_{t}$ of the Hilbert function in degree $t$ is:

(i) $\left(\begin{array}{c}t+2 \\ 2\end{array}\right)$ if $t<3 m$;

(ii) $n\left(\begin{array}{c}m+1 \\ 2\end{array}\right)-s(t, n, m)$ if $t \geq n m / 3$; and

(iii) $\left(\begin{array}{c}t+2 \\ 2\end{array}\right)-\left(\begin{array}{c}t-3 r+2 \\ 2\end{array}\right)+n\left(\begin{array}{c}m-r+1 \\ 2\end{array}\right)-s(t-3 r, n, m-r)$ if $n>9$ and $3 m \leq t<m n / 3$, where $r=\lceil(m n-3 t) /(n-9)\rceil$.

Proof. This result is a corollary of the main result of [12]. Let $F=t L-m E_{1}-\cdots-$ $m E_{n}$ with respect to the morphism $\pi: Y \rightarrow \mathbf{P}^{2}$ obtained by blowing up the points $p_{i}$.

Let $C^{\prime} \subset Y$ be the proper transform of $C$ with respect to $\pi$. Since the points $p_{i}$ blown up are smooth points on $C$, we see $\left[C^{\prime}\right]=\left[3 L-E_{1}-\cdots-E_{n}\right]$ (and hence $C^{\prime}$ is an anticanonical divisor). Moreover, each component of $C^{\prime}$ is the proper transform $D^{\prime}$ of a component $D$ of $C$, and each of the components of $C$ (and hence of $C^{\prime}$ ) is reduced. (To see this note that $n_{D}>0$ for each component $D$ of $C$ since the points $p_{i}$ are evenly distributed, but the number of points $p_{i}$ which lie on $D$ is $n_{D}$ and all of the points $p_{i}$ are smooth points of $C$, so each component of $C$ has a smooth point and hence must be reduced.)

In addition, the following statements are equivalent:

(a) $F \cdot D^{\prime} \geq 0$ for every irreducible component $D^{\prime}$ of $C^{\prime}$;

(b) $F \cdot C^{\prime} \geq 0$; and

(c) $F \cdot D^{\prime} \geq 0$ for some irreducible component $D^{\prime}$ of $C^{\prime}$.

Clearly, (a) implies (b), and (b) implies (c). We now show that (c) implies (a). If $C^{\prime}$ has only one component, then (c) and (a) are trivially equivalent, so suppose $D_{1}^{\prime}$ and $D_{2}^{\prime}$ are distinct components of $C^{\prime}$. In order to show that $F \cdot D_{1}^{\prime} \geq 0$ implies 
$F \cdot D_{2}^{\prime} \geq 0$, we will use the assumption that the points $p_{i}$ are evenly distributed smooth points of $C$. Let $D_{j}=\pi\left(D_{j}^{\prime}\right)$, so $D_{j}^{\prime}$ is the proper transform of $D_{j}$. Because the points are evenly distributed, we have $n_{D_{j}}=n\left(\operatorname{deg}\left(D_{j}\right)\right) / 3$. Thus $n_{D_{j}}$ of the $n$ points $p_{i}$ lie on $D_{j}$. Because the points are smooth points of $C$, we have $\left[D_{j}^{\prime}\right]=$ $\left[\operatorname{deg}\left(D_{j}\right) L-\sum_{p_{i} \in D_{j}} E_{i}\right]$, where the sum involves $n_{D_{j}}$ terms. Thus $F \cdot D_{j}^{\prime} \geq 0$ can be rewritten as $t \operatorname{deg}\left(D_{j}\right)-m n_{D_{j}} \geq 0$. Substituting $n\left(\operatorname{deg}\left(D_{j}\right)\right) / 3$ for $n_{D_{j}}$ gives $t \operatorname{deg}\left(D_{j}\right)-m n\left(\operatorname{deg}\left(D_{j}\right)\right) / 3 \geq 0$ which is equivalent to $3 t-m n \geq 0$, which is itself just $F \cdot C^{\prime} \geq 0$. Thus $F \cdot D_{1}^{\prime} \geq 0$ and $F \cdot D_{2}^{\prime} \geq 0$ are both equivalent to $F \cdot C^{\prime} \geq 0$, and hence $F \cdot D_{1}^{\prime} \geq 0$ if and only if $F \cdot D_{2}^{\prime} \geq 0$. This shows (c) implies (a).

We now show that $h^{0}\left(Y, \mathcal{O}_{Y}(F)\right)=0$ if and only if $t<3 m$. For $t \geq 3 m$, we have $\mathcal{O}_{Y}(F)=\mathcal{O}_{Y}\left((t-3 m) L+m C^{\prime}\right)$, and hence $h^{0}\left(Y, \mathcal{O}_{Y}(F)\right)>0$. If, however, $t<3 m$, then $3 t<9 m \leq n m$ so $F \cdot C^{\prime}<0$, and hence, as we saw above, $F \cdot D^{\prime}<0$ for each component $D^{\prime}$ of $C^{\prime}$, in which case each component $D^{\prime}$ of $C^{\prime}$ is a fixed component of $|F|$ so $h^{0}\left(Y, \mathcal{O}_{Y}(F)\right)=h^{0}\left(Y, \mathcal{O}_{Y}\left(F-C^{\prime}\right)\right)=h^{0}\left(Y, \mathcal{O}_{Y}\left((t-3) L-(m-1) E_{1}-\right.\right.$ $\left.\left.\cdots-(m-1) E_{n}\right)\right)$. But $t-3<3(m-1)$, so, by the same argument, we can again subtract off $C^{\prime}$ without changing $h^{0}$. Continuing in this way we eventually obtain $h^{0}\left(Y, \mathcal{O}_{Y}(F)\right)=h^{0}\left(Y, \mathcal{O}_{Y}((t-3 m) L)\right)=h^{0}\left(\mathbb{P}^{2}, \mathcal{O}_{\mathbb{P}^{2}}(t-3 m)\right)$, but $h^{0}\left(\mathbb{P}^{2}, \mathcal{O}_{\mathbb{P}^{2}}(t-\right.$ $3 m))=0$ since $t-3 m<0$. Thus $h_{Z}(t)=\left(\begin{array}{c}t+2 \\ 2\end{array}\right)$ for $t<3 m$, which proves (i).

Next consider (ii). If $t \geq n \mathrm{~m} / 3$, i.e., if $F \cdot C^{\prime} \geq 0$, then as we saw above $F \cdot D^{\prime} \geq 0$ for every component $D^{\prime}$ of $C^{\prime}$. But as we also saw above, $(t-3 m) L+m C^{\prime} \in|F|$, hence $F$ is nef. If $t>n m / 3$ (in which case $s(t, n, m)=0$ ), then $F \cdot C^{\prime}>0$, so by [12, Theorem III.1 (a,b)], $h^{1}\left(Y, \mathcal{O}_{Y}(F)\right)=0$. Thus (1.2) gives $h_{Z}(t)=n\left(\begin{array}{c}m+1 \\ 2\end{array}\right)=$ $n\left(\begin{array}{c}m+1 \\ 2\end{array}\right)-s(t, n, m)$ as claimed. We are left with the case that $t=n m / 3$.

Suppose $t=n m / 3$ and $n=9$. Thus $F=m C^{\prime}$ and $F \cdot C^{\prime}=0$ (because $n=9$ and $t=3 m)$, so $\left(C^{\prime}\right)^{2}=0$. By duality we have $h^{2}\left(Y, \mathcal{O}_{Y}\left(m C^{\prime}\right)\right)=h^{0}\left(Y, \mathcal{O}_{Y}(-(m+\right.$ 1) $\left.\left.C^{\prime}\right)\right)=0$, so by Riemann-Roch we have $h^{0}\left(Y, \mathcal{O}_{Y}(F)\right)-h^{1}\left(Y, \mathcal{O}_{Y}(F)\right)=1+$ $\left(F^{2}+C^{\prime} \cdot F\right) / 2=1$. Since $F$ is nef, so is $i C^{\prime}$ for all $i \geq 0$. Since $F \cdot C^{\prime}=0$, either $|F|$ has an element disjoint from $C^{\prime}$ or $F$ and $C^{\prime}$ share a common component.

If $|F|$ has an element disjoint from $C^{\prime}$, then $\mathcal{O}_{C^{\prime}}(F)$ is trivial, so $h^{0}\left(C^{\prime}, \mathcal{O}_{C^{\prime}}(F)\right)=$ 1 since $C$ (and hence $C^{\prime}$ ) is connected and reduced. Suppose $F$ and $C^{\prime}$ share a common component. Then $C^{\prime}$ is in the base locus of $|F|$ by [12, Corollary III.2], and hence $h^{0}\left(Y, \mathcal{O}_{Y}(F)\right)=h^{0}\left(Y, \mathcal{O}_{Y}\left(F-C^{\prime}\right)\right)$. Let $\phi$ be the least $i>0$ (possibly infinite) such that $C^{\prime}$ is not in the base locus of $\left|i C^{\prime}\right|$. Then we have that $h^{0}\left(Y, \mathcal{O}_{Y}\left(j C^{\prime}\right)\right)=h^{0}\left(Y, \mathcal{O}_{Y}\left((j+1) C^{\prime}\right)\right)$ for $0 \leq j<\phi-1$, so by induction (using the base case $h^{0}\left(Y, \mathcal{O}_{Y}\right)=1$ and the fact $\left.h^{0}\left(Y, \mathcal{O}_{Y}\left(j C^{\prime}\right)\right)-h^{1}\left(Y, \mathcal{O}_{Y}\left(j C^{\prime}\right)\right)=1\right)$ we have $h^{0}\left(Y, \mathcal{O}_{Y}\left(j C^{\prime}\right)\right)=1$ and $h^{1}\left(Y, \mathcal{O}_{Y}\left(j C^{\prime}\right)\right)=0$ for all $0 \leq j<\phi$. It follows that

$$
0 \rightarrow \mathcal{O}_{Y}\left((s-1) C^{\prime}\right) \rightarrow \mathcal{O}_{Y}\left(s C^{\prime}\right) \rightarrow \mathcal{O}_{C^{\prime}}\left(s C^{\prime}\right) \rightarrow 0
$$

is exact on global sections for $1 \leq s \leq \phi$, and that $h^{0}\left(C^{\prime}, \mathcal{O}_{C^{\prime}}\left(s C^{\prime}\right)\right)=0$ and $h^{1}\left(C^{\prime}, \mathcal{O}_{C^{\prime}}\left(s C^{\prime}\right)\right)=0$ for $0<s<\phi$. Thus $\mathcal{O}_{C^{\prime}}\left(s C^{\prime}\right)$ is nontrivial for $0<s<\phi$. Since for all $m,\left|m C^{\prime}\right|$ either has an element disjoint from $C^{\prime}$ or $C^{\prime}$ is in the base locus of $\left|m C^{\prime}\right|$, we see that $\mathcal{O}_{C^{\prime}}\left(\phi C^{\prime}\right)$ is trivial, and hence $\phi$ is the order of $\mathcal{O}_{C^{\prime}}\left(C^{\prime}\right)$ in 
$\operatorname{Pic}\left(C^{\prime}\right)$. But since the points $p_{i}$ blown up are smooth points of $C$, the morphism $\pi$ : $Y \rightarrow \mathbb{P}^{2}$ induces an isomorphism $C \rightarrow C^{\prime}$, and under this isomorphism, $\mathcal{O}_{C}(3 L) \otimes$ $\mathcal{O}_{C}\left(-p_{1}-\cdots-p_{n}\right)$ corresponds to $\mathcal{O}_{C^{\prime}}\left(C^{\prime}\right)$, so we see $\phi=\lambda$. It follows that $h^{0}\left(C^{\prime}, \mathcal{O}_{C^{\prime}}\left(s C^{\prime}\right)\right)=h^{1}\left(C^{\prime}, \mathcal{O}_{C^{\prime}}\left(s C^{\prime}\right)\right)$ for all $s \geq 0$, and these are both 1 if $\mathcal{O}_{C^{\prime}}\left(s C^{\prime}\right)$ is trivial (i.e., if $s$ is a multiple of $\lambda$ ) and they are 0 otherwise.

We now claim that $(\star)$ is exact on global sections for all $s \geq 1$. It is enough to show this when $s$ is a multiple of $\lambda$, because otherwise, as we noted above, $h^{0}\left(C^{\prime}, \mathcal{O}_{C^{\prime}}\left(s C^{\prime}\right)\right)$ equals 0 and hence $(\star)$ is automatically exact on global sections. But $\left|\lambda C^{\prime}\right|$ (and hence also $\left|i \lambda C^{\prime}\right|$ for all $\left.i \geq 1\right)$ has an element disjoint from $C^{\prime}$, so $H^{0}\left(Y, \mathcal{O}_{Y}\left(i \lambda C^{\prime}\right)\right) \rightarrow$ $H^{0}\left(C^{\prime}, \mathcal{O}_{C^{\prime}}\left(i \lambda C^{\prime}\right)\right)$ is onto, which shows that $(\star)$ is exact on global sections when $s$ is a multiple of $\lambda$.

It follows that $(\star)$ is also exact on $h^{1}$ 's (since as above $h^{2}\left(Y, \mathcal{O}_{Y}\left(i C^{\prime}\right)\right)=0$ for all $i \geq 0)$, and hence that $h^{1}\left(Y, \mathcal{O}_{Y}\left(m C^{\prime}\right)\right)=h^{1}\left(Y, \mathcal{O}_{Y}\right)+\sum_{1 \leq i \leq m} h^{1}\left(C^{\prime}, \mathcal{O}_{C^{\prime}}\left(i C^{\prime}\right)\right)$. Now $h^{1}\left(Y, \mathcal{O}_{Y}\right)=h^{1}\left(\mathbb{P}^{2}, \mathcal{O}_{\mathbb{P}^{2}}\right)=0$ and $h^{1}\left(C^{\prime}, \mathcal{O}_{C^{\prime}}\left(i C^{\prime}\right)\right)$ is 1 if and only if $i$ is a multiple of $\lambda$ and it is 0 otherwise. Thus $h^{1}\left(Y, \mathcal{O}_{Y}\left(m C^{\prime}\right)\right)$ is the number of summands $h^{1}\left(C^{\prime}, \mathcal{O}_{C^{\prime}}\left(i C^{\prime}\right)\right)$ for which $i$ is a multiple of $\lambda$; i.e., $h^{1}\left(Y, \mathcal{O}_{Y}\left(m C^{\prime}\right)\right)=\lfloor m / \lambda\rfloor$, which is just $s(t, n, m)$. This implies that $h_{Z}(t)=n\left(\begin{array}{c}m+1 \\ 2\end{array}\right)-s(t, n, m)$, as claimed.

If $t=n m / 3$ but $n>9$, then $F^{2}>0$ so by [12, Theorem III.1 (c)] either $\mathcal{O}_{C^{\prime}}(F)$ is trivial (in which case $s(t, n, m)=1$ ) and $h^{1}\left(Y, \mathcal{O}_{Y}(F)\right)=1$ (and hence $h_{Z}(t)=$ $\left.n\left(\begin{array}{c}m+1 \\ 2\end{array}\right)-1=n\left(\begin{array}{c}m+1 \\ 2\end{array}\right)-s(t, n, m)\right)$, or $C^{\prime}$ is in the base locus of $|F|$. If $C^{\prime}$ is in the base locus, then by [12, Theorem III.1(d)] and the fact that $F^{2}>0$ we have $\mathcal{O}_{C^{\prime}}(F)$ is not trivial (in which case $s(t, n, m)=0$ ) and $h^{1}\left(Y, \mathcal{O}_{Y}(F)\right)=0$, and hence $h_{Z}(t)=n\left(\begin{array}{c}m+1 \\ 2\end{array}\right)-s(t, n, m)$, as claimed.

Now consider case (iii); i.e., $3 m \leq t<n m / 3$ and $n>9$. Then $F \cdot D^{\prime}<0$ for each component $D^{\prime}$ of $C^{\prime}$ (since the points are evenly distributed), in which case $h^{0}\left(Y, \mathcal{O}_{Y}(F)\right)=h^{0}\left(Y, \mathcal{O}_{Y}\left(F-C^{\prime}\right)\right)=h^{0}\left(Y, \mathcal{O}_{Y}\left((t-3) L-(m-1) E_{1}-\cdots-\right.\right.$ $\left.\left.(m-1) E_{n}\right)\right)$. If $t-3<n(m-1) / 3$, we can subtract $C^{\prime}$ off again. This continues until we have subtracted $C^{\prime}$ off $r=\lceil(m n-3 t) /(n-9)\rceil$ times, at which point we have that $F-r C^{\prime}$ is nef (and hence $t-3 r \geq n(m-r) / 3$ ) and effective and $h^{0}\left(Y, \mathcal{O}_{Y}(F)\right)=h^{0}\left(Y, \mathcal{O}_{Y}\left(F-r C^{\prime}\right)\right)$. Applying (ii) to $F-r C^{\prime}$ gives $\left(\begin{array}{c}t-3 r+2 \\ 2\end{array}\right)-$ $h^{0}\left(Y, \mathcal{O}_{Y}\left(F-r C^{\prime}\right)\right)=h_{(m-r) X}(t-3 r)=n\left(\begin{array}{c}m-r+1 \\ 2\end{array}\right)-s(t-3 r, n, m-r)$ or $h^{0}\left(Y, \mathcal{O}_{Y}\left(F-r C^{\prime}\right)\right)=\left(\begin{array}{c}t-3 r+2 \\ 2\end{array}\right)-\left(n\left(\begin{array}{c}m-r+1 \\ 2\end{array}\right)-s(t-3 r, n, m-r)\right)$. Substituting this in for $h^{0}\left(Y, \mathcal{O}_{Y}(F)\right)$ in $h_{Z}(t)=\left(\begin{array}{c}t+2 \\ 2\end{array}\right)-h^{0}\left(Y, \mathcal{O}_{Y}(F)\right)$ gives (iii).

Remark 4.3. We can now write down all possible Hilbert functions for $n \geq 9$ points of multiplicity $m$ for each possible choice of Hilbert function for the reduced scheme given by the points, if the points are smooth points of a reduced cubic curve and evenly distributed. Suppose $X=p_{1}+\cdots+p_{n}$ and $m=1$. If 3 does not divide $n$, or it does but $s(n / 3, n, 1)=0$, then the difference function for the Hilbert function of $X$ is the same as given in (3.2), but if 3 divides $n$ and $s(n / 3, n, 1)=1$, then $X$ is a complete intersection and the difference function for the Hilbert function of $X$ is the same as given in (3.1). 
We now compare our results for $Z=2 X=2\left(p_{1}+\cdots+p_{n}\right)$ with those obtained in Proposition 3.1 and Theorem 3.4, and we explicitly list those cases skipped there (because there we assumed $n=3 t$ with $t \geq 3$ in Proposition 3.1 and $n=3 t+\delta$ with $t>5-\delta$ in Theorem 3.4).

Say $n \equiv 1 \bmod 3$. Then the difference function for the Hilbert function is:

$n=10: 123456630$

$n=13: 12345666420$, and for

$n=10+3 x$ for $x>1$ : the result is the same as given in Theorem 3.4(i).

Next, say $n \equiv 2 \bmod 3$. Then the difference function for the Hilbert function is:

$n=11: 1234566510$, and for

$n=11+3 x$ for $x>0$ : the result is the same as given in Theorem 3.4(i).

If $n=3 x$, there are two possibilities. If $s(2 x, n, 2)=0$ for the given points (i.e., the divisor $2 p_{1}+\cdots+2 p_{n}$ on $C$ is not cut out by a curve of degree $2 x$, or equivalently $\mathcal{O}_{C}\left(2 x L-2 E_{1}-\cdots-2 E_{n}\right)$ is not trivial), then the difference function for the Hilbert function is:

$n=9: 12345660$, and

$n=3 x: 123456 \ldots 63 \ldots 30$ for $x \geq 4$, where the number of 6 's is $x-1$ and the number of trailing 3's is $x-3$. For $x>5$, this is the same as the result given in Theorem 3.4 (ii).

If $s(2 x, n, 2)=1$ for the given points (i.e., the divisor $2 p_{1}+\cdots+2 p_{n}$ on $C$ is cut out by a curve of degree $2 x$, or equivalently $\mathcal{O}_{C}\left(2 x L-2 E_{1}-\cdots-2 E_{n}\right)$ is trivial), but $s(n / 3, n, 1)=0$ (so $p_{1}+\cdots+p_{n}$ is not cut out by a curve of degree $x$, which is equivalent to saying that $\mathcal{O}_{C}\left(x L-E_{1}-\cdots-E_{n}\right)$ is not trivial $)$, then the difference function for the Hilbert function is:

$n=9: 123456510$,

$n=12: 12345666210$, and

$n=3 x: 123456 \ldots 63 \ldots 3210$ for $x>4$, where the number of 6's is $x-1$ and the number of trailing 3's is $x-4$. For $x>5$, this is the same as the result given in Theorem 3.4 (ii).

Now say $n=3 x$ and $s(x, n, 1)=1$. In this case, $X$ is the complete intersection of $C$ and a form of degree $t$, and the difference function for the Hilbert function of $2 X$ is:

$n=9: 123456420$

$n=12: 12345665310$ and

$n=3 x$ for $x>4$ : the result is the same as given in Proposition 3.1.

Remark 4.4. The possible values of the Hilbert functions as given in Theorem 4.2 depend partly on what torsion occurs in $\operatorname{Pic}(C)$, and this in turn is affected by the characteristic of $k$. When $C$ is smooth, see [14, Example IV.4.8.1] for a discussion of the torsion. When $C$ is reduced but not smooth, the torsion is easy to understand since it is all contained in the identity component $\operatorname{Pic}^{0}(C)$ of $\operatorname{Pic}(C)$, whose group structure is isomorphic either to the additive or multiplicative groups of the ground 
field. (See for example [13, Proposition 5.2], which states a result for curves of socalled canonical type. But for any reduced cubic $C$, one can always find a set of 9 evenly distributed smooth points of $C$, and the proper transform $C^{\prime}$ with respect to blowing those points up is a curve of canonical type, meaning that $C^{\prime} \cdot D=K_{X} \cdot D=0$ for every component $D$ of $C^{\prime}$. Since the points blown up are smooth on $C, C$ and $C^{\prime}$ are isomorphic and thus so $\operatorname{are} \operatorname{Pic}(C)$ and $\operatorname{Pic}\left(C^{\prime}\right)$, hence the conclusion of [13, Proposition 5.2] applies to $C$, even though $C$ is not itself of canonical type.) When $C$ is reduced and irreducible but singular, for example, the result is that $\operatorname{Pic}^{0}(C)$ is the additive group of the ground field when $C$ is cuspidal and it is the multiplicative group of the field when $C$ is nodal [14, Exercise II.6.9]. In particular, if $C$ is an irreducible cuspidal cubic curve over a field of characteristic zero, then $\operatorname{Pic}^{0}(C)$ is torsion free, so $h_{2 X}$ cannot be $(1,2,3,4,5,6,6,6,2,1)$; indeed, this follows, after a simple calculation, because if $\mathcal{O}_{C}\left(2 x L-2 p_{1}-\cdots-2 p_{n}\right)$ is trivial, then so is $\mathcal{O}_{C}\left(x L-p_{1}-\cdots-p_{n}\right)$. On the other hand, $\mathcal{O}_{C}\left(x L-p_{1}-\cdots-p_{n}\right)$ can be nontrivial even if $\mathcal{O}_{C}\left(2 x L-2 p_{1}-\cdots-2 p_{n}\right)$ is trivial if the characteristic is 2 or if the singular point is a node but the characteristic is not 2 , since in those cases $\operatorname{Pic}(C)$ has elements of order 2 .

Remark 4.5. We can also use the method of proof of Theorem 4.2 to recover the result of Theorem 3.7 for the Hilbert function of $m X=m\left(p_{1}+\cdots+p_{n}\right)$ for $n \geq 9$ points on a reduced, irreducible cubic curve $C$ where $p_{1}$, say, is the singular point and $m$ is 1 or 2. As is now clear, the approach of Theorem 4.2 is to determine $h^{0}\left(Y, \mathcal{O}_{Y}(t L-\right.$ $\left.\left.m E_{1}-\cdots-m E_{n}\right)\right)$ for all $t$, and then translate this into the Hilbert function or the difference function for $m X$.

This translation is purely mechanical and the resulting Hilbert functions in the case that $n \geq 12$ are already given in Theorem 3.7 (we leave writing down the Hilbert functions for $9 \leq n \leq 11$ using the results that follow as an exercise for the reader). Thus it is the calculation of $h^{0}\left(Y, \mathcal{O}_{Y}\left(t L-m E_{1}-\cdots-m E_{n}\right)\right)$ that is of most interest, and it is on this that we now focus.

Let $Y$ be the blow up of the points, let $C^{\prime}$ be the proper transform of $C$, and let $F_{t}=t L-E_{1}-\cdots-E_{n}$ and $G_{t}=t L-2\left(E_{1}+\cdots+E_{n}\right)$, where we denote by $L$ both a general line in the plane and its pullback to $Y$. Up to linear equivalence, note that $C^{\prime}=3 L-2 E_{1}-E_{2}-\cdots-E_{n}$.

The goal here is to compute the values of $h^{0}\left(Y, \mathcal{O}_{Y}\left(F_{t}\right)\right)$ and $h^{0}\left(Y, \mathcal{O}_{Y}\left(G_{t}\right)\right)$. For $t<3$, Bézout tells us that $h^{0}\left(X, \mathcal{O}_{Y}\left(F_{t}\right)\right)=0$, since $F_{t} \cdot C^{\prime}<0$ (hence $h^{0}\left(Y, \mathcal{O}_{Y}\left(F_{t}\right)\right)$ equals $h^{0}\left(Y, \mathcal{O}_{Y}\left(F_{t}-C^{\prime}\right)\right)$ and $\left(F_{t}-C^{\prime}\right) \cdot L<0$ (hence $h^{0}\left(Y, \mathcal{O}_{Y}\left(F_{t}-C^{\prime}\right)\right)=0$ ). If $t \geq 3$, then certainly $h^{0}\left(Y, \mathcal{O}_{Y}\left(F_{t}\right)\right)>0$, since $F_{t}=(t-3) L+C^{\prime}+E_{1}$. We consider three cases, according to whether $F_{t} \cdot C^{\prime}<0, F_{t} \cdot C^{\prime}>0$ or $F_{t} \cdot C^{\prime}=0$.

If $0>F_{t} \cdot C^{\prime}=3 t-2-(n-1)$ (i.e., if $\left.3 \leq t<(n+1) / 3\right)$, then $h^{0}\left(Y, \mathcal{O}_{Y}\left(F_{t}\right)\right)=$ $h^{0}\left(Y, \mathcal{O}_{Y}\left(F_{t}-C^{\prime}\right)\right)=h^{0}\left(Y, \mathcal{O}_{Y}\left((t-3) L+E_{1}\right)\right)=h^{0}\left(Y, \mathcal{O}_{Y}((t-3) L)\right)=$ $h^{0}\left(\mathbb{P}^{2}, \mathcal{O}_{\mathbb{P}^{2}}((t-3) L)\right)=\left(\begin{array}{c}t-3+2 \\ 2\end{array}\right)$, since $F_{t}-C^{\prime}=(t-3) L+E_{1}$. If $F_{t} \cdot C^{\prime}>0$ (i.e., $t>(n+1) / 3)$, then $h^{0}\left(Y, \mathcal{O}_{Y}\left(F_{t}\right)\right)=\left(\begin{array}{c}t+2 \\ 2\end{array}\right)-n$ (since $F_{t}$, meeting both components 
of $-K_{Y}=C^{\prime}+E_{1}$ positively, is nef and hence $h^{1}\left(Y, \mathcal{O}_{Y}\left(F_{t}\right)\right)=0$ by [12, Theorem III.1 (a,b)]).

This leaves the case that $t=(n+1) / 3$. This means that $\mathcal{O}_{C^{\prime}}\left(F_{t}\right)$ has degree 0 . Consider the exact sequence

$$
0 \rightarrow \mathcal{O}_{Y}\left((t-3) L+E_{1}\right) \rightarrow \mathcal{O}_{Y}\left(F_{t}\right) \rightarrow \mathcal{O}_{C^{\prime}}\left(F_{t}\right) \rightarrow 0
$$

By an analogous argument to the one used to show $h^{1}\left(Y, \mathcal{O}_{Y}\left(F_{t}\right)\right)=0$ when $t>$ $(n+1) / 3$, we obtain that $h^{1}\left(\mathcal{O}_{Y}\left((t-3) L+E_{1}\right)\right)=0$. But $C^{\prime}$ is a smooth rational curve, so also the third sheaf in the sequence has vanishing first cohomology. Thus we obtain $h^{1}\left(Y, O_{Y}\left(F_{t}\right)\right)=0$, hence the points impose independent conditions. It follows that $h^{0}\left(Y, O_{Y}\left(F_{t}\right)\right)=\left(\begin{array}{c}t+2 \\ 2\end{array}\right)-n$ also for $t=(n+1) / 3$.

We thus have: $h^{0}\left(Y, \mathcal{O}_{Y}\left(F_{t}\right)\right)=0$ for $0 \leq t<3 ; h^{0}\left(Y, \mathcal{O}_{Y}\left(F_{t}\right)\right)=\left(\begin{array}{c}t-1 \\ 2\end{array}\right)$ for $3 \leq t<(n+1) / 3$; and $h^{0}\left(Y, \mathcal{O}_{Y}\left(F_{t}\right)\right)=\left(\begin{array}{c}t+2 \\ 2\end{array}\right)-n$ for $t \geq(n+1) / 3$.

A similar analysis works for $2 X$. There are now four ranges of degrees. The first range is $t<6$, in which case $h^{0}\left(Y, \mathcal{O}_{Y}\left(G_{t}\right)\right)=0$ by Bézout, arguing as above. For $t \geq 6$, we have $h^{0}\left(Y, \mathcal{O}_{Y}\left(G_{t}\right)\right)>0$, since up to linear equivalence we have $G_{t}=(t-6) L+2\left(C^{\prime}+E_{1}\right)$. The second range is now $6 \leq t<(n+8) / 3$; in this case $2 C^{\prime}$ is, by Bézout, a fixed component of $\left|G_{t}\right|$, so $h^{0}\left(Y, \mathcal{O}_{Y}\left(G_{t}\right)\right)=h^{0}\left(Y, \mathcal{O}_{Y}((t-\right.$ 6) $\left.\left.L+2 E_{1}\right)\right)=\left(\begin{array}{c}t-4 \\ 2\end{array}\right)$. The third range is $(n+8) / 3 \leq t<(2 / 3)(n+1)$, for which $C^{\prime}$ is a fixed component of $\left|G_{t}\right|$ (and $G_{t}-C^{\prime}=(t-6) L+C^{\prime}+2 E_{1}$ is nef) so $h^{0}\left(Y, \mathcal{O}_{Y}\left(G_{t}\right)\right)=h^{0}\left(Y, \mathcal{O}_{Y}\left(G_{t}-C^{\prime}\right)\right)$ and we know $h^{0}\left(Y, \mathcal{O}_{Y}\left(G_{t}-C^{\prime}\right)\right)$ by Theorem 4.2 (ii) if $n>9$, while $h^{1}\left(Y, \mathcal{O}_{Y}\left(G_{t}-C^{\prime}\right)\right)=0$ by [12, Theorem III.1 (a,b)]) if $n=9$, so again we know $h^{0}\left(Y, \mathcal{O}_{Y}\left(G_{t}-C^{\prime}\right)\right)$. The last range is $t \geq(2 / 3)(n+1)$, in which case $G_{t}$ is nef. If $t>(2 / 3)(n+1)$, then $G_{t}$ meets $-K_{Y}$ positively, so $h^{1}\left(Y, \mathcal{O}_{Y}\left(G_{t}\right)\right)=0\left[12\right.$, Theorem III.1 (a,b)]), and $h^{0}\left(Y, \mathcal{O}_{Y}\left(G_{t}\right)\right)=\left(\begin{array}{c}t+2 \\ 2\end{array}\right)-3 n$. We are left with the case that $t=(2 / 3)(n+1)$. Consider the exact sequence

$$
0 \rightarrow \mathcal{O}_{Y}\left((t-3) L-E_{2}-\cdots-E_{n}\right) \rightarrow \mathcal{O}_{Y}\left(G_{t}\right) \rightarrow \mathcal{O}_{C^{\prime}}\left(G_{t}\right) \rightarrow 0
$$

Since $G_{t} \cdot C^{\prime} \geq 0$ and $C^{\prime}$ is smooth and rational, we have $h^{1}\left(C^{\prime}, \mathcal{O}_{C^{\prime}}\left(G_{t}\right)\right)=0$, and since $\mathcal{O}_{Y}\left((t-3) L-E_{2}-\cdots-E_{n}\right)=\mathcal{O}_{Y}\left((t-6) L+C^{\prime}+2 E_{1}\right)$ and $(t-6) L+C^{\prime}+2 E_{1}$ is nef (as observed above) with $\left(G_{t}-C^{\prime}\right) \cdot C^{\prime}>0$, we have $h^{1}\left(Y, \mathcal{O}_{Y}\left(G_{t}-C^{\prime}\right)\right)=0$ [12, Theorem III.1 (a,b)]) and hence $h^{1}\left(Y, \mathcal{O}_{Y}\left(G_{t}\right)\right)=0$, so in fact $h^{0}\left(Y, \mathcal{O}_{Y}\left(G_{t}\right)\right)=$ $\left(\begin{array}{c}t+2 \\ 2\end{array}\right)-3 n$.

Remark 4.6. Here we comment on what is left to do if one wants to recover the results of Section 2 using the methods of Section 4. So consider $n=9$ points on a given cubic $C$ (but note that there may be more than one cubic through the points), either all of multiplicity 1 or all of multiplicity 2 . The case that the points are evenly distributed smooth points of $C$ is done above, as is the case that the curve $C$ is reduced and irreducible. The case that the points all lie on a conic follows from the known result for configuration types of points on a conic [7]. What is left is that the points do not all 
lie on any given conic (and hence $C$ is reduced) and either: one or more of the points is not a smooth point of $C$ and $C$ is not irreducible, or the points are not distributed evenly (and hence again $C$ is not irreducible). The four reducible cubics that arise are: a conic and a line tangent to the conic; a conic and a transverse line; three lines passing through a point; and three lines with no point common to all three. Each of these cases leads to a number of cases depending on how the points are placed (such as how many are on each component and whether one or more is a singular point of the cubic, but also depending on the group law of the cubic). Analyzing these cases would give a complete result of the Hilbert functions of the form $h_{X}$ and $h_{2 X}$ for a reduced scheme $X$ consisting of 9 distinct points of the plane.

Acknowledgments. We thank Z. Teitler for helpful comments and the referee for a careful reading of the paper.

\section{Bibliography}

[1] Bigatti, A., Geramita, A. V., Migliore, J., Geometric consequences of extremal behavior in a theorem of Macaulay. Trans Amer Math Soc. 1994;346:203-235.

[2] Buckles, M., Guardo, E., Van Tuyl, A., Fat points on a generic almost complete intersection. Matematiche (Catania). 2000;55:191-202.

[3] Buckles, M., Guardo, E., Van Tuyl, A., Fat points on a grid in $\mathbb{P}^{2}$. Matematiche (Catania). 2000;55:169-189.

[4] Davis, E., 0-dimensional subschemes of $\mathbb{P}^{2}$ : new application of Castelnuovo's function. Ann Univ Ferrara Sez VII Sci Mat. 1986;XXXII:93-107.

[5] Davis, E., Geramita, A. V., Orecchia, F., Gorenstein algebras and the Cayley-Bacharach theorem. Proc Amer Math Soc. 1985;93:593-597.

[6] Ellingsrud, G., Sur le schéma de Hilbert des variétés de codimension 2 dans $\mathbb{P}^{e}$ à cône de Cohen-Macaulay. Ann Sci Éc Norm Supér (4) 1975;8:423-431.

[7] Geramita, A. V., Harbourne, B., Migliore, J., Classifying Hilbert functions of fat point subschemes in $\mathbb{P}^{2}$. Collect Math. 2009;60(2):159-192.

[8] Geramita, A. V., Maroscia, P., Roberts, L., The Hilbert function of a reduced $k$-algebra. J Lond Math Soc (2). 1983;28:443-452.

[9] Geramita, A. V., Migliore, J., Sabourin, L., On the first infinitesimal neighborhood of a linear configuration of points in $\mathbb{P}^{2}$. J Algebra. 2006;298(2):563-611.

[10] Guardo, E., Van Tuyl, A., Some results on fat points whose support is a complete intersection minus a point. In: Projective varieties with unexpected properties. De Gruyter; 2005. p. 257-266.

[11] Guardo, E., Van Tuyl, A., Powers of complete intersections: graded Betti numbers and applications. Illinois J Math. 2005;49:265-279. 
[12] Harbourne, B., Anticanonical rational surfaces. Trans Amer Math Soc. 1997;349:11911208.

[13] Harbourne, B., Lang, W. E., Multiple fibres on rational elliptic surfaces. Trans Amer Math Soc. 1988;307:205-223.

[14] Hartshorne, R., Algebraic Geometry. vol. 52 of Graduate texts in mathematics. New York: Springer-Verlag; 1977.

[15] Migliore, J., Introduction to liaison theory and deficiency modules. vol. 165 of Progress in mathematics. Birkhäuser; 1998.

\section{Author Information}

Anthony V. Geramita, Dipartimento di Matematica, Università di Genova, Genova, Italia and Department of Mathematics, Queen's University, Kingston, ON, Canada.

E-mail: anthony.geramita@gmail.com

Brian Harbourne, Department of Mathematics, University of Nebraska, Lincoln, NE, USA.

E-mail: bharbour@math.unl.edu

Juan C. Migliore, Department of Mathematics, University of Notre Dame, South Bend, IN, USA.

E-mail: migliore.1@nd.edu 
